\title{
Dynamics of shielding of a moving charged particle in a confined electron plasma
}

\author{
Andrey Elizarov* and Vladimir Litvinenko ${ }^{\dagger}$ \\ Collider-Accelerator Department, Brookhaven National Laboratory, \\ Upton, New York 11973, USA and Department of Physics and Astronomy, \\ Stony Brook University, Stony Brook, New York 11794, USA
}

(Received 29 July 2014; published 9 April 2015)

\begin{abstract}
Dynamics of shielding of a moving charged particle in a confined plasma, which represents a realistic model of an electron beam in accelerators, is studied. This is a longstanding problem in plasma physics with important applications in accelerator physics. However, only solutions for an infinite unrealistic plasma are available. We developed a novel method to solve this problem, which consists of transformation of the Vlasov-Poisson differential equations to an integral equation for the Laplace image of the electron density perturbation created by an external charge. The integral equation is then solved numerically via the piecewise polynomial collocation method and the fast Fourier transform. We present thorough analysis of the results obtained and their physical interpretation.
\end{abstract}

DOI: 10.1103/PhysRevSTAB.18.044001

PACS numbers: 52.40.Mj, 52.30.-q, 52.27.Aj, 52.65.Ff

\section{INTRODUCTION}

Recent progress in hadron beam cooling techniques has triggered an interest to computation of the dynamics of shielding of a moving charged particle, the test charge problem, in an electron beam. In the modulator section of the coherent electron cooling $(\mathrm{CeC})$ [1], information about a hadron is recorded as a density perturbation in a co-propagating electron beam. Then, this perturbation is amplified in a free electron laser (FEL) [2,3], and thereafter, the amplified perturbation creates an electric field that accelerates or decelerates the hadron depending on its velocity deviation. Precise computation of the dynamics of shielding of a hadron in an electron beam is required to provide a theoretical description of $\mathrm{CeC}$ device and obtain the correct parameters' values for the facility currently under construction at Brookhaven National Laboratory [4].

The simplest formulation of the test charge problem is an evaluation of screening of a stationary particle in an infinite plasma (by plasma we mean a collisionless single-species electron plasma), this is the well-known Debye screening. For a particle moving in an infinite plasma, it is possible to solve the corresponding equations, i.e., the Vlasov-Poisson system, via the Laplace and Fourier transforms [5-7]. For the 1D infinite plasma with the Cauchy equilibrium velocity distribution, the exact solution can be obtained [6]. For the 3D infinite plasma with the Lorentz equilibrium

\footnotetext{
*andrey.elizarov@stonybrook.edu

†v@bnl.gov
}

Published by the American Physical Society under the terms of the Creative Commons Attribution 3.0 License. Further distribution of this work must maintain attribution to the author $(s)$ and the published article's title, journal citation, and DOI. velocity distribution, the solution can be expressed as a one-dimensional integral [5], and for other cases, the integral transforms must be inverted numerically [6].

An infinite plasma is an unrealistic model of an electron beam. Many effects are very different in finite and infinite plasmas and so the shielding, as we shall see at the end of this paper. For the first time, the Vlasov-Poisson equations for a finite beam with the microcanonical equilibrium distribution was considered by Gluckstern [8]; then these ideas were further developed by him and Venturini in [9]. In these papers, they studied a beam's response to an external magnetic field of a special form. However, plasma with the microcanonical equilibrium distribution does not represent a realistic model of a physical system and they did not study shielding of an external charge. There also have been some considerations of a test charge problem for a semi-infinite bounded plasma [10].

In the present article, we develop a method for computation of shielding of a charged particle in a confined plasma. We define confined plasma as plasma with an integrable equilibrium distribution, at least over some spatial coordinates. An example of such plasma is an electron beam with finite emittance along these coordinates. There are two cases of practical interest: a fully confined plasma (its spatial distribution is integrable over all coordinates), e.g., a ball in 3D space with the normal (Maxwell) spatial distribution, which is a realistic model of an electron bunch in a bunched beam, and a partially confined plasma (its spatial distribution is integrable over some coordinates), e.g., a longitudinally infinite electron beam with the finite transverse emittance. Although, with some modifications, the method can be applied to infinite and partially confined plasmas, we will focus on a fully confined case, since this model is more realistic. 
We start with the formulation of the problem. Then we describe two different methods, which are similar, but one is less general and more effective numerically than the other. They both lead to the Fredholm integral equation of the second type for the Laplace image of the perturbation's density. We call such equations the Laplace-Fredholm equations for the density perturbation. Then, we review the piecewise polynomial collocation method (PPCM) for the Fredholm integral equation, and describe our numerical method for the Laplace-Fredholm equation that utilizes the PPCM and the discrete Fourier transform. Thereafter, we present our numerical results for shielding of a charged particle in the $1 \mathrm{D}, 2 \mathrm{D}$, and 3D plasmas with the normal (Maxwell) spatial and velocity distributions. We compare our results to those obtained previously for the infinite plasma [6] and provide physical interpretation of the results. We then discuss application of these results to the $\mathrm{CeC}$ device now being constructed at Brookhaven National Laboratory.

\section{THE TEST CHARGE PROBLEM FOR A CONFINED PLASMA}

An interaction of a collisionless anisotropic plasma with an external charge with an electron density $d(\vec{x}, t)$ in a center-of-momentum frame of the plasma can be described by the Vlasov-Poisson [11] system of equations:

$$
\begin{gathered}
\frac{\partial f}{\partial t}+\vec{v} \cdot \frac{\partial f}{\partial \vec{x}}+\frac{d \vec{p}}{d t} \cdot \frac{\partial f}{\partial \vec{p}}=0, \quad f \equiv f(\vec{x}, \vec{v}, t), \\
\vec{v}=\frac{\partial H}{\partial \vec{p}}, \quad \frac{d \vec{p}}{d t}=-\frac{\partial H}{\partial \vec{x}}, \quad H=H_{0}+e U(\vec{x}, t), \\
H \equiv H(\vec{x}, \vec{p}, t), \quad H_{0} \equiv H_{0}(\vec{x}, \vec{p}), \\
H_{0}=\sum_{i, j} x_{i} \alpha_{i j} x_{j}+\sum_{i, j} p_{i} \beta_{i j} p_{j} \equiv \vec{x} \cdot \hat{\alpha} \vec{x}+\vec{p} \cdot \hat{\beta} \vec{p}, \\
\frac{\partial^{2}}{\partial \vec{x}^{2}} U(\vec{x}, t)=-\frac{e}{\epsilon_{0}}\left(n_{1}(\vec{x}, t)+d(\vec{x}, t)\right),
\end{gathered}
$$

where the dynamics of the plasma's phase-space electron density $f(\vec{x}, \vec{v}, t)$ is governed by the Vlasov equation (1), and the electric potential $U(\vec{x}, t)$ - by the Poisson equation (5); the charge density can be obtained by multiplying the electron density by an electron charge $e$ : $\rho(\vec{x}, t)=e n(\vec{x}, t) \cdot n_{1}(\vec{x}, t)$ is a plasma's electron density perturbation, caused by an interaction with the external charge, that we are going to evaluate; it is related to the plasma's phase-space electron density perturbation $f_{1}(\vec{x}, \vec{v}, t)$ via

$$
n_{1}(\vec{x}, t)=\int f_{1}(\vec{x}, \vec{v}, t) d \vec{v} .
$$

Also, we employ the Hamilton's equations (2), in which $H$ is a total Hamiltonian that includes unperturbed equilibrium Hamiltonian $H_{0}$ and the contribution $e U(\vec{x}, t)$, caused by the external charge and the plasma's electron density perturbation. In the unperturbed Hamiltonian $H_{0}$, the second term is a kinetic energy and $\hat{\beta}$ characterizes the plasma's anisotropy, while the first term is responsible for spatial confining of the plasma. In accelerator physics, $\hat{\alpha}$ is determined by the focusing magnetic fields in an accelerator. We consider the problem with a moving point charge with a density

$$
d(\vec{x}, t)=-Z \delta(\vec{x}-\vec{y}(t))
$$

where $\vec{y}(t)$ is its trajectory; we assume that the trajectory is unaffected by the space charge fields.

The Vlasov-Poisson system (1)-(5) differs from the one for the infinite plasma [6] only by the $\hat{\alpha}$-term in $H_{0}$. This term makes $\frac{\partial H_{0}}{\partial \vec{x}}$ nonzero and an extra term appears in the Vlasov equation, which makes it unsolvable by the methods that worked for the infinite plasma.

We consider the diagonal matrices in the unperturbed Hamiltonian $H_{0}$ :

$$
\alpha_{i j}=\alpha_{i} \delta_{i j}, \quad \beta_{i j}=\beta_{i} \delta_{i j},
$$

where $\delta_{i j}$ is the Kronecker delta. In this case,

$$
\begin{gathered}
H_{0}=\sum_{i} \alpha_{i} x_{i}^{2}+\sum_{i} \beta_{i} p_{i}^{2}, \\
\vec{v}=\frac{\partial H}{\partial \vec{p}}, \quad v_{i}=2 \beta_{i} p_{i}, \quad \vec{v}=\hat{\gamma} \vec{p}, \\
\text { where } \gamma_{i j}=\frac{\partial v_{j}}{\partial p_{i}}=2 \beta_{i} \delta_{i j},
\end{gathered}
$$

and,

$$
\frac{d \vec{p}}{d t}=-\frac{\partial H}{\partial \vec{x}}, \quad-\frac{\partial H_{0}}{\partial x_{i}}=-2 \alpha_{i} x_{i}, \quad \text { or } \quad-\frac{\partial H_{0}}{\partial \vec{x}}=-\hat{\eta} \vec{x},
$$

where $\eta_{i j}=2 \alpha_{i} \delta_{i j}$. Thus, we have

$$
H_{0}=\sum_{i} \alpha_{i} x_{i}^{2}+\sum_{i} \frac{v_{i}^{2}}{4 \beta_{i}} .
$$

Introducing new notations 


$$
a_{i}=\alpha_{i}, \quad b_{i}=\frac{1}{4 \beta_{i}},
$$

we rewrite the unperturbed Hamiltonian in a form:

$$
H_{0}=\sum_{i} a_{i} x_{i}^{2}+\sum_{i} b_{i} v_{i}^{2}
$$

For the diagonal matrices, we have

$$
\gamma_{i j}=\gamma_{i} \delta_{i j}, \quad \eta_{i j}=\eta_{i} \delta_{i j}, \quad \frac{\partial f}{\partial \vec{p}}=\hat{\gamma} \frac{\partial f}{\partial \vec{v}} .
$$

For a fully confined symmetric $1 \mathrm{D}, 2 \mathrm{D}$, or 3D plasmas, we have:

$$
\begin{array}{ll}
\alpha_{i}=\alpha, & \beta_{i}=\frac{1}{2 m_{0}}, \quad b_{i}=\frac{m_{0}}{2}, \\
\gamma_{i}=\frac{1}{m_{0}}, & \eta_{i}=2 \alpha .
\end{array}
$$

All our considerations are applicable to the 1D, 2D, and 3D spaces. The only differences are in the expressions for the Green's function for the Poisson's equation (5) and in the units of the dimensional constants. These aspects are discussed in Secs. III D and III F, respectively.

\section{INTEGRAL EQUATION FOR THE TEST CHARGE PROBLEM}

In the present section, we describe how the VlasovPoisson (1)-(5) system can be transformed into the Fredholm integral equation of the second type for the Laplace image of the density perturbation $n_{1}(\vec{x}, t)$. We use the method of unperturbed orbits to solve the linearized Vlasov equation, and then insert into the obtained equation an expression for the potential in the form of an integral of the Green's function multiplied by the electron density and $\frac{e}{\epsilon_{0}}$. We derived two different integral equations. The first one is for general orbits; the second is for periodic orbits only (for the fully confined plasma), but it involves integrals that can be evaluated faster. We describe these two methods in order.

\section{A. Method for general orbits}

We represent the phase-space electron density $f$, $f \equiv f\left(\vec{x}, \vec{v}, t_{1}\right)$, in a form $f=f_{0}+f_{1}$, where $f_{0}, f_{0} \equiv$ $f_{0}(\vec{x}, \vec{v})$ is an equilibrium electron density and $f_{1}$, $f_{1} \equiv f_{1}\left(\vec{x}, \vec{v}, t_{1}\right)$, is the unknown electron density perturbation, which satisfies the linearized Vlasov equation:

$$
\frac{\partial f_{1}}{\partial t_{1}}+\vec{v} \cdot \frac{\partial f_{1}}{\partial \vec{x}}-\frac{\partial H_{0}}{\partial \vec{x}} \hat{\gamma} \frac{\partial f_{1}}{\partial \vec{v}}=e \frac{\partial U}{\partial \vec{x}} \hat{\gamma} \frac{\partial f_{0}}{\partial \vec{v}},
$$

where we denoted the time variable as $t_{1}$ just for future convenience and introduced the notation:

$$
\vec{a} \hat{b} \vec{c} \equiv \sum_{i} a_{i} b_{i i} c_{i}=\vec{a} \cdot \hat{b} \vec{c}
$$

where $\hat{b}$ is a diagonal matrix. The third term in Eq. (18) represents interaction of the perturbation with the focusing fields and the term in the right-hand side represents interaction of the unknown perturbation and external charge with the equilibrium space-charge filed; it will be clear from (25). After substituting

$$
\vec{x}=\vec{X}_{0}\left(t_{1}\right), \quad \vec{v}=\vec{V}_{0}\left(t_{1}\right),
$$

where $\vec{X}_{0}\left(t_{1}\right), \vec{V}_{0}\left(t_{1}\right)$ are the unperturbed orbits, the solutions of the following Hamilton's system:

$$
\dot{\vec{X}}_{0}\left(t_{1}\right)=\vec{V}_{0}\left(t_{1}\right), \quad \dot{\vec{V}}_{0}\left(t_{1}\right)=-\hat{\gamma} \frac{\partial H_{0}}{\partial \vec{X}_{0}\left(t_{1}\right)}
$$

with the initial conditions:

$$
\vec{X}_{0}(t)=\vec{x}, \quad \vec{V}_{0}(t)=\vec{v},
$$

into Eq. (18), terms in its left-hand side will be equal to a full time derivative of $f_{1}$ :

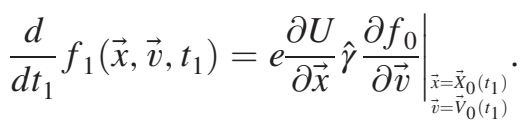

With a reasonable initial condition $f_{1}(\vec{x}, \vec{v}, 0)=0$, this equation can be integrated:

$$
f_{1}(\vec{x}, \vec{v}, t)=\left.e \int_{0}^{t} \frac{\partial U}{\partial \vec{x}} \hat{\gamma} \frac{\partial f_{0}}{\partial \vec{v}}\right|_{\substack{\vec{x}=\vec{x}_{0}\left(t_{1}\right) \\ \vec{v}=\bar{V}_{0}\left(t_{1}\right)}} d t_{1} .
$$

When plasma oscillations are considered [12], an equation analogous to (24) is already a solution, since in this case, $U$ is a known quantity and does not depend on $f_{1}$. In our case, this is not a solution yet, since $U$ depends on $f_{1}$.

Assuming boundary conditions at infinity, we have for the electric potential:

$$
\begin{aligned}
U\left(\vec{x}, t_{1}\right) & =U_{1}\left(\vec{x}, t_{1}\right)+U_{2}\left(\vec{x}, t_{1}\right) \\
& =\frac{e}{\epsilon_{0}} \int n_{1}\left(\vec{x}^{\prime}, t_{1}\right) G\left(\vec{x}, \vec{x}^{\prime}\right) d \vec{x}^{\prime}-Z \frac{e}{\epsilon_{0}} G\left(\vec{x}, \vec{Y}\left(t_{1}\right)\right),
\end{aligned}
$$

where $G\left(\vec{x}, \vec{x}^{\prime}\right)$ is the Green's function for the Poisson equation, $U_{1}(\vec{x})$ is a potential of the plasma's electron density perturbation, and $U_{2}(\vec{x})$ is a potential of the ion and $\vec{Y}\left(t_{1}\right)$ is its trajectory. Inserting (26) into (24) and integrating over $\vec{v}$, we obtain 


$$
n_{1}(\vec{x}, t)=\left.\frac{e^{2}}{\epsilon_{0}} \int_{0}^{t} \int n_{1}\left(\vec{x}^{\prime}, t_{1}\right) \int \frac{\partial G\left(\vec{x}, \vec{x}^{\prime}\right)}{\partial \vec{x}} \hat{\gamma} \frac{\partial f_{0}}{\partial \vec{v}}\right|_{\substack{\vec{x}=\vec{X}_{0}\left(t_{1}\right) \\ \vec{v}=\vec{V}_{0}\left(t_{1}\right)}} d \vec{v} d \vec{x}^{\prime} d t_{1}+\left.e \int_{0}^{t} \int \frac{\partial U_{2}\left(\vec{x}, t_{1}\right)}{\partial \vec{x}} \hat{\gamma} \frac{\partial f_{0}}{\partial \vec{v}}\right|_{\substack{\vec{x}=\vec{X}_{0}\left(t_{1}\right) \\ \vec{v}=\vec{V}_{0}\left(t_{1}\right)}} d \vec{v} d t_{1}
$$

The first term in the right-hand side can be considered as a convolution over time, since the unperturbed orbits are functions of $\left(t-t_{1}\right)$, as we shall show in Sec. III B. Applying the Laplace transform over $t$, and taking into account that the Laplace image of a convolution of two functions equals a product of their Laplace images, we obtain

$$
N_{1}(\vec{x}, s)=\left.\frac{e^{2}}{\epsilon_{0}} \int N_{1}\left(\vec{x}^{\prime}, s\right) \mathcal{L} \int \frac{\partial G\left(\vec{x}, \vec{x}^{\prime}\right)}{\partial \vec{x}} \hat{\gamma} \frac{\partial f_{0}}{\partial \vec{v}}\right|_{\substack{\vec{x}=\vec{x}_{0}(0) \\ \vec{v}=\vec{V}_{0}(0)}} d \vec{v} d \vec{x}^{\prime}+\left.e \mathcal{L} \int_{0}^{t} \int \frac{\partial U_{2}\left(\vec{x}, t_{1}\right)}{\partial \vec{x}} \hat{\gamma} \frac{\partial f_{0}}{\partial \vec{v}}\right|_{\substack{\vec{x}=\vec{x}_{0}\left(t_{1}\right) \\ \vec{v}=\vec{v}_{0}\left(t_{1}\right)}} d \vec{v} d t_{1}
$$

where $\mathcal{L}$ is a Laplace transform operator and

$$
N_{1}(\vec{x}, s) \equiv \mathcal{L} n_{1}(\vec{x}, t)
$$

is a Laplace image of the unknown function $n_{1}(\vec{x}, t)$. Equation (28) is the Fredholm integral equation of the second type with a kernel

$$
K\left(\vec{x}, \vec{x}^{\prime}, s\right)=\left.\mathcal{L} \int \frac{\partial G\left(\vec{x}, \vec{x}^{\prime}\right)}{\partial \vec{x}} \hat{\gamma} \frac{\partial f_{0}}{\partial \vec{v}}\right|_{\substack{\vec{x}=\vec{x}_{0}(0) \\ \vec{v}=\vec{v}_{0}(0)}} d \vec{v}
$$

and a left-hand side

$$
F(\vec{x}, s)=-\left.Z \frac{e^{2}}{\epsilon_{0}} \mathcal{L} \int_{0}^{t} \int \frac{\partial G\left(\vec{x}, \vec{Y}\left(t_{1}\right)\right)}{\partial \vec{x}} \hat{\gamma} \frac{\partial f_{0}}{\partial \vec{v}}\right|_{\substack{\vec{x}=\vec{x}_{\left(t t_{1}\right.} \\ \vec{v}=\vec{V}_{0}\left(t_{1}\right)}} d \vec{v} d t_{1}
$$

With these notations, the equation can be written in a standard form:

$$
F(\vec{x}, s)=N_{1}(\vec{x}, s)-\lambda \int N_{1}\left(\vec{x}^{\prime}, s\right) K\left(\vec{x}, \vec{x}^{\prime}, s\right) d \vec{x}^{\prime}
$$

where $\lambda=\frac{e^{2}}{\epsilon_{0}}$.

\section{B. Unperturbed orbits}

In some cases, the Hamilton's equations (21) with the initial conditions (22) can be solved exactly. For example, for the Hamiltonian (13), we have

$$
\begin{aligned}
& X_{0 i}\left(t_{1}\right)=x_{i} \cos \left(\omega_{i}\left(t-t_{1}\right)\right)-\frac{v_{i}}{\omega_{i}} \sin \left(\omega_{i}\left(t-t_{1}\right)\right), \\
& V_{0 i}\left(t_{1}\right)=v_{i} \cos \left(\omega_{i}\left(t-t_{1}\right)\right)+x_{i} \omega_{i} \sin \left(\omega_{i}\left(t-t_{1}\right)\right)
\end{aligned}
$$

where $\omega_{i}=\sqrt{\gamma_{i} \eta_{i}}$. The Hamilton's system (21) with the initial conditions (22) defines a map from the phase space to the space of trajectories: $(\vec{x}, \vec{v}, t) \mapsto\left(\dot{\vec{X}}_{0}(\cdot), \dot{\vec{V}}_{0}(\cdot)\right)$. We call the orbits (33), (34) elliptical; and spherical, if all $\omega_{i}$ are equal.

When $H_{0}$ does not depend on $\vec{x}$, all $\omega_{i}$ equal zero, and we obtain orbits for the infinite plasma:

$$
\begin{gathered}
X_{0 i}\left(t_{1}\right)=x_{i}-v_{i}\left(t-t_{1}\right), \\
V_{0 i}\left(t_{1}\right)=v_{i}
\end{gathered}
$$

For an electron beam finite in the transverse direction, and infinite in longitudinal, we have the orbits (33), (34) for the transverse directions and (35), (36) for the longitudinal one. We will call the unperturbed orbits simply orbits or trajectories.

\section{Method for periodic orbits}

In this subsection, we describe a method suitable for the periodic orbits with the same periods in all dimensions, spherical orbits fall into this category. Instead of integrating from 0 to $t$, as in (24), for the case of periodic trajectories, we can integrate the linearized Vlasov equation from $t$ to $t+\frac{2 \pi}{\omega}$, where $\frac{2 \pi}{\omega}$ is the orbits' period. We start with the linearized Vlasov equation (18), as before:

$$
\frac{\partial f_{1}}{\partial t_{1}}+\vec{v} \cdot \frac{\partial f_{1}}{\partial \vec{x}}=\frac{\partial H_{0}}{\partial \vec{x}} \hat{\gamma} \frac{\partial f_{1}}{\partial \vec{v}}+e \frac{\partial U}{\partial \vec{x}} \hat{\gamma} \frac{\partial f_{0}}{\partial \vec{v}}
$$

then, taking into account

$\mathcal{L} \frac{\partial f_{1}\left(t_{1}\right)}{\partial t_{1}}=s F_{1}(s)-f_{1}(0), \quad$ in our case $f_{1}(0)=0$

where $f_{1}\left(t_{1}\right) \equiv f_{1}$ and $F_{1}$ is the Laplace image of $f_{1}$, $F_{1} \equiv F_{1}(s) \equiv F_{1}(\vec{x}, \vec{v}, s)$, we do the Laplace transform of the equation and then multiply it by $\mathrm{e}^{t_{1} s}$ : 


$$
\begin{aligned}
& s F_{1} \mathrm{e}^{t_{1} s}+\vec{v} \cdot \frac{\partial F_{1}}{\partial \vec{x}} \mathrm{e}^{t_{1} s} \\
& =\frac{\partial H_{0}}{\partial \vec{x}} \hat{\gamma} \frac{\partial F_{1}}{\partial \vec{v}} \mathrm{e}^{t_{1} s}+e \frac{\partial \bar{U}}{\partial \vec{x}} \hat{\gamma} \frac{\partial f_{0}}{\partial \vec{v}} \mathrm{e}^{t_{1} s} .
\end{aligned}
$$

Taking into account that

$$
\frac{\partial}{\partial t_{1}}\left(F_{1}(s) \mathrm{e}^{t_{1} s}\right)=s F_{1}(s) \mathrm{e}^{t_{1} s}
$$

we obtain

$$
\begin{aligned}
& \frac{\partial}{\partial t_{1}}\left(F_{1}(s) \mathrm{e}^{t_{1} s}\right)+\vec{v} \cdot \frac{\partial}{\partial \vec{x}}\left(F_{1}(s) \mathrm{e}^{t_{1} s}\right) \\
& \quad=\frac{\partial H_{0}}{\partial \vec{x}} \hat{\gamma} \frac{\partial}{\partial \vec{v}}\left(F_{1}(s) \mathrm{e}^{t_{1} s}\right)+e \frac{\partial\left(\bar{U}(s) \mathrm{e}^{t_{1} s}\right)}{\partial \vec{x}} \hat{\gamma} \frac{\partial f_{0}}{\partial \vec{v}}
\end{aligned}
$$

then, doing substitution

$$
\vec{x}=\vec{X}_{0}\left(t_{1}\right), \quad \vec{v}=\vec{V}_{0}\left(t_{1}\right),
$$

where $\vec{X}_{0}\left(t_{1}\right), \vec{V}_{0}\left(t_{1}\right)$ are the unperturbed orbits, we obtain

$$
\begin{aligned}
& \frac{d}{d t_{1}}\left(F_{1}\left(\vec{X}_{0}\left(t_{1}\right), \vec{V}_{0}\left(t_{1}\right), s\right) \mathrm{e}^{t_{1} s}\right) \\
& =\left.e \frac{\partial\left(\bar{U}(\vec{x}, s) \mathrm{e}^{t_{1} s}\right)}{\partial \vec{x}} \hat{\gamma} \frac{\partial f_{0}(\vec{x}, \vec{v})}{\partial \vec{v}}\right|_{\substack{\vec{x} \vec{X}_{0}\left(t_{1}\right) \\
\vec{v}=\vec{V}_{0}\left(t_{1}\right)}} .
\end{aligned}
$$

Then we integrate this equation over $t_{1}$ :

$$
F_{1}\left(\vec{X}_{0}\left(t+\frac{2 \pi}{\omega}\right), \vec{V}_{0}\left(t+\frac{2 \pi}{\omega}\right), s\right) \mathrm{e}^{\left(t+\frac{2 \pi}{\omega}\right) s}-F_{1}\left(\vec{X}_{0}(t), \vec{V}_{0}(t), s\right) \mathrm{e}^{t s}=\left.e \int_{t}^{t+\frac{2 \pi}{\omega}} \frac{\partial\left(\bar{U}(\vec{x}, s) \mathrm{e}^{t_{1} s}\right)}{\partial \vec{x}} \hat{\gamma} \frac{\partial f_{0}(\vec{x}, \vec{v})}{\partial \vec{v}}\right|_{\substack{\vec{x}=\vec{X}_{0}\left(t_{1}\right) \\ \vec{v}=V_{0}\left(t_{1}\right)}} d t_{1}
$$

where $\bar{U}(\vec{x}, s)$ is the Laplace image of the potential. Using periodicity of the trajectories and initial conditions (22), we obtain

$$
\begin{aligned}
F_{1}(\vec{x}, \vec{v}, s)= & e \frac{\mathrm{e}^{-t s}}{\mathrm{e}^{\frac{2 \pi}{\omega} s}-1} \\
& \times\left.\int_{t}^{t+\frac{2 \pi}{\omega}} \frac{\partial \bar{U}(\vec{x}, s)}{\partial \vec{x}} \hat{\gamma} \frac{\partial f_{0}(\vec{x}, \vec{v})}{\partial \vec{v}}\right|_{\substack{\vec{x}=\vec{X}_{0}\left(t_{1}\right) \\
\vec{v}=\bar{v}_{0}\left(t_{1}\right)}} \mathrm{e}^{t_{1} s} d t_{1},
\end{aligned}
$$

where $t$ is some free constant that can be set to any number, $t$ is also present in trajectories, as it is evident from (33) and (34); for example, it can be set to 0 without any loss of generality. For the potential, we use expressions (25), (26) and obtain for $\bar{U}(\vec{x}, s)$ :

$$
\begin{gathered}
\bar{U}(\vec{x}, s)=\bar{U}_{1}(\vec{x}, s)+\bar{U}_{2}(\vec{x}, s) \\
=\frac{e}{\epsilon_{0}} \int N_{1}\left(\vec{x}^{\prime}, s\right) G\left(\vec{x}, \vec{x}^{\prime}\right) d \vec{x}^{\prime}-\frac{e Z}{\epsilon_{0}} \mathcal{L}_{t_{2}, s} G\left(\vec{x}, \vec{Y}\left(t_{2}\right)\right) .
\end{gathered}
$$

Inserting this into Eq. (45) and integrating it over $\vec{v}$, we obtain the same equation as in the previous case:

$$
F(\vec{x}, s)=N_{1}(\vec{x}, s)-\lambda \int N_{1}\left(\vec{x}^{\prime}, s\right) K\left(\vec{x}, \vec{x}^{\prime}, s\right) d \vec{x}^{\prime},
$$

but with the different expressions for the kernel and left-hand side:

$$
\begin{gathered}
K\left(\vec{x}, \vec{x}^{\prime}, s\right)=\left.\frac{\mathrm{e}^{-t s}}{\mathrm{e}^{\frac{2 \pi}{\omega} s}-1} \iint_{t}^{t+\frac{2 \pi}{\omega}} \frac{\partial G\left(\vec{x}, \vec{x}^{\prime}\right)}{\partial \vec{x}} \hat{\gamma} \frac{\partial f_{0}(\vec{x}, \vec{v})}{\partial \vec{v}}\right|_{\substack{\vec{x}=\vec{X}_{0}\left(t_{1}\right) \\
\vec{v}=\vec{V}_{0}\left(t_{1}\right)}} \mathrm{e}^{t_{1} s} d t_{1} d \vec{v}, \\
F(\vec{x}, s)=-\left.Z \frac{e^{2}}{\epsilon_{0}} \frac{\mathrm{e}^{-t s}}{\mathrm{e}^{\frac{2 \pi}{\omega} s}-1} \iint_{0}^{\infty} \int_{t}^{t+\frac{2 \pi}{\omega}} \frac{\partial G\left(\vec{x}, \vec{Y}\left(t_{2}\right)\right)}{\partial \vec{x}} \hat{\gamma} \frac{\partial f_{0}(\vec{x}, \vec{v})}{\partial \vec{v}}\right|_{\substack{\vec{x}=\vec{X}_{0}\left(t_{1}\right) \\
\vec{v}=\vec{v}_{0}\left(t_{1}\right)}} \mathrm{e}^{\left(t_{1}-t_{2}\right) s} d t_{1} d t_{2} d \vec{v},
\end{gathered}
$$

and $\lambda=\frac{e^{2}}{\epsilon_{0}}$. 


\section{Introducing dimensionless variables}

The dimensionless units that we define for the confined plasma slightly differ from the ones that we used for the infinite one [6]. There, we used the following dimensionless variables:

$$
\begin{gathered}
\overrightarrow{\mathrm{x}}=\frac{\vec{x}}{r_{\mathrm{D}}}, \quad \overrightarrow{\mathrm{v}}=\frac{\vec{v}}{v_{\mathrm{rms}}}, \quad \mathrm{t}=\frac{t}{t_{\mathrm{p}}} \equiv t \omega_{\mathrm{p}}, \\
\overrightarrow{\mathrm{k}}=\vec{k} r_{\mathrm{D}}, \quad \mathrm{s}=\frac{s}{\omega_{\mathrm{p}}} \equiv s t_{\mathrm{p}},
\end{gathered}
$$

where we used sans-serif font to denote them, and the root mean square velocity $v_{\text {rms }}$, plasma frequency $\omega_{\mathrm{p}}$, plasma period $t_{\mathrm{p}}$, and Debye radius $r_{\mathrm{D}}$ were defined as follows

$$
\begin{gathered}
v_{\mathrm{rms}}=\sqrt{\frac{1}{\rho} \int v^{2} f_{0}(\vec{v}) d \vec{v}}, \quad \omega_{\mathrm{p}}=\sqrt{\frac{e^{2} \rho}{m_{0} \epsilon_{0}}}=\frac{1}{t_{\mathrm{p}}}, \\
r_{\mathrm{D}}=\frac{v_{\mathrm{rms}}}{\omega_{\mathrm{p}}}=v_{\mathrm{rms}} t_{\mathrm{p}} .
\end{gathered}
$$

The vacuum permittivity $\epsilon_{0}$ has different units in the $1 \mathrm{D}$, $2 \mathrm{D}$, and $3 \mathrm{D}$ cases, i.e.,

$$
\left[\epsilon_{0}\right]=\frac{C^{2} T^{2}}{L^{d} M}
$$

where $d$ is a dimensionality of the space, and $C, T, L$, and $M$ are the charge, time, length, and mass units, respectively. And we used the dimensionless equilibrium density $f_{0}(\vec{v})$ defined via:

$$
f_{0}(\vec{v})=\rho f_{d} \mathbf{f}_{0}(\overrightarrow{\mathbf{v}}),
$$

where $[\rho]=[n(\vec{x}, t)]=L^{-d}$, and $\left[f_{d}\right]=\left[v_{\mathrm{rms}}\right]^{-d}$.

For the confined plasma, the equilibrium distribution also depends on $\vec{x}$, thus we have

$$
f_{0}(\vec{x}, \vec{v})=\rho f_{d} \mathrm{f}_{0}(\overrightarrow{\mathbf{x}}, \overrightarrow{\mathrm{v}}), \quad[\rho]=1, \quad\left[f_{d}\right]=\left[v_{\mathrm{rms}} r_{\mathrm{D}}\right]^{-d} .
$$

In this case, $\rho$ has different units; we redefine plasma frequency accordingly:

$$
\omega_{\mathrm{p}}=\sqrt{\frac{e^{2} \rho r_{\mathrm{D}}^{-d}}{m_{0} \epsilon_{0}}} .
$$

In Sec III G, we rewrite the integral equations for the test charge problem using these units. In the next subsection, we detail the equilibrium distributions.

\section{E. Equilibrium distributions}

Since any function of the unperturbed Hamiltonian is a solution of the unperturbed Vlasov equation, we consider equilibrium distributions that are functions of the unperturbed Hamiltonian, i.e., $f_{0}\left(H_{0}\right)$. For $H_{0}$, we have

$$
H_{0}=\sum_{i} a_{i} x_{i}^{2}+\sum_{i} b_{i} v_{i}^{2}
$$

We consider the normal and Cauchy distributions. The latter was very useful for the infinite plasma, since it has a simpler density, and hence, the computations were faster. We normalize the distributions such that

$$
\int f_{0}(\vec{x}, \vec{v}) d \vec{x} d \vec{v}=\rho .
$$

\section{Normal distribution}

For the normal distribution, we have

$$
f(\vec{x}, \vec{v})=\rho \frac{\left(\prod_{i} a_{i} b_{i}\right)^{\frac{1}{2}}}{H_{c}^{d} \pi^{d}} \mathrm{e}^{-\sum_{i} \frac{a_{i}}{H_{c}} x_{i}^{2}-\sum_{i} \frac{b_{i}}{H_{c}} v_{i}^{2}}
$$

and we obtain for $v_{\text {rms }}^{2}$ via (53):

$$
v_{\mathrm{rms}}^{2}=\frac{H_{c}}{2} \frac{\sum_{j=1}^{d} \prod_{i=1, i \neq j}^{d} b_{i}}{\prod_{i=1}^{d} b_{i}}
$$

or, for the isotropic case $\left(b_{i}=b\right.$, for all $\left.i\right)$,

$$
v_{\mathrm{rms}}^{2}=\frac{H_{c}}{2} \frac{d}{b}
$$

We will not use this $v_{\mathrm{rms}}$, though; rather, we define $v_{\mathrm{rms}, i}$ for each spatial dimension, it turns out that they are given by:

$$
v_{\mathrm{rms}, i}=\sqrt{\frac{H_{c}}{2 b_{i}}},
$$

and, for the isotropic plasma, we have

$$
v_{\mathrm{rms}}=\sqrt{\frac{H_{c}}{2 b}} .
$$

While it is possible to consider general anisotropic case, this leads to more dimensionless parameters in the dimensionless density; thus, for simplicity, we consider isotropic case with the Hamiltonian

$$
\mathrm{H}_{0}=\sum_{i} \mathrm{a}_{i} \mathrm{x}_{i}^{2}+\frac{1}{2} \sum_{i} \mathrm{v}_{i}^{2}
$$


We have for the distribution:

$$
\mathrm{f}_{0}(\overrightarrow{\mathrm{x}}, \overrightarrow{\mathrm{v}})=\frac{1}{\pi^{d}} \mathrm{e}^{-\sum_{i} \mathrm{a}_{i} \mathrm{x}_{i}^{2}-\frac{1}{2} \sum_{i} v_{i}^{2}}
$$

the conventions we used here are slightly different from those used in [6], and

$$
f_{d}=\left(\frac{b}{H_{c}}\right)^{\frac{d}{2}} \prod_{i} \sqrt{\frac{a_{i}}{H_{c}}} .
$$

Alternatively, we can consider $v_{\mathrm{rms}}=\sqrt{\frac{H_{c}}{b}}$, this gives

$$
\mathrm{f}_{0}(\overrightarrow{\mathrm{x}}, \overrightarrow{\mathrm{v}})=\frac{1}{\pi^{d}} \mathrm{e}^{-\sum_{i} \mathrm{a}_{i} \mathrm{x}_{i}^{2}-\sum_{i} \mathrm{v}_{i}^{2}}
$$

by setting $\mathrm{a}_{i}=1$ for all $i$ and integrating over $\overrightarrow{\mathrm{x}}$, this distribution can be reduced to the same velocity distribution that we considered in [6] for the infinite plasma. We have for the derivative:

$$
\frac{\partial \mathrm{f}_{0}(\overrightarrow{\mathbf{x}}, \overrightarrow{\mathrm{v}})}{\partial \overrightarrow{\mathrm{v}}}=-2 \overrightarrow{\mathrm{v}} \mathrm{f}_{0}(\overrightarrow{\mathbf{x}}, \overrightarrow{\mathrm{v}})
$$

In next subsections, we will need plasma's spatial size. Integrating the distribution (69) over $\vec{v}$, we obtain

$$
\int \mathrm{f}_{0}(\overrightarrow{\mathbf{x}}, \overrightarrow{\mathrm{v}}) d \overrightarrow{\mathrm{v}}=\frac{1}{\pi^{\frac{d}{2}}} \mathrm{e}^{-\sum_{i} \mathrm{a}_{i} \mathrm{x}_{i}^{2}}
$$

The variance of this integrated distribution in a certain direction $j$ is

$$
\sigma_{j}^{2}=\frac{1}{2}\left(\prod_{i} \mathrm{a}_{i}\right)^{-\frac{1}{2}} \frac{1}{\mathrm{a}_{j}},
$$

It is well known that approximately $99.9 \%$ of the values of the 1D, 2D, and 3D normally distributed random variables lie within a "four sigma" interval. Thus, we assume that the plasma with the normal spatial distribution lies within a closed interval $\left[-4 \sigma_{j}, 4 \sigma_{j}\right]$ in dimension $j$. If we assume that for all $j, \mathrm{a}_{j}=1$, all $\sigma_{j}$ equal $2^{-\frac{1}{2}} \approx 0.707$ and $4 \sigma_{j} \approx 2.83$ for all $j$. For the case when all $\sigma_{j}$ are equal, we introduce $\sigma$ :

$$
\sigma=\sqrt{2} \sigma_{j},
$$

which equals one, when all $\mathrm{a}_{j}$ equal one.

\section{Cauchy distribution}

All of the considerations for the normal distribution also can be applied to other distributions. For example, for the Cauchy distribution, we have

$$
\begin{aligned}
f_{0}(\vec{x}, \vec{v})= & \rho \frac{\left(\prod_{i} a_{i} b_{i}\right)^{\frac{1}{2}}}{H_{c}^{d} \pi^{d}} \frac{\Gamma\left(\frac{1+d}{2}\right)}{\Gamma\left(\frac{1}{2}\right) \pi^{\frac{d}{2}}} \\
& \times\left(1+\sum_{i} \frac{a_{i}}{H_{c}} x_{i}^{2}+\sum_{i} \frac{b_{i}}{H_{c}} v_{i}^{2}\right)^{-\frac{1+d}{2}},
\end{aligned}
$$

where $\Gamma(z)$ is the gamma function [13]; in this case, $v_{\text {rms }}$ cannot be computed, since the corresponding integral is divergent; we use

$$
v_{\mathrm{rms}}=\sqrt{\frac{H_{c}}{b}} .
$$

Considering an isotropic plasma $\left(b_{i}=b\right.$, for all $\left.i\right)$, we obtain

$$
\mathrm{f}_{0}(\overrightarrow{\mathbf{x}}, \overrightarrow{\mathbf{v}})=\frac{\Gamma\left(\frac{1+d}{2}\right)}{\Gamma\left(\frac{1}{2}\right) \pi^{\frac{d}{2}}} \frac{1}{\left(1+\sum_{i} \mathrm{a}_{i} \mathrm{x}_{i}^{2}+\sum_{i} \mathrm{v}_{i}^{2}\right)^{\frac{1+d}{2}}},
$$

and

$$
f_{d}=\left(\sqrt{\frac{b}{H_{c}}}\right)^{d} \prod_{i} \sqrt{\frac{a_{i}}{H_{c}}}
$$

and for the derivative:

$$
\frac{\partial \mathrm{f}_{0}(\overrightarrow{\mathrm{x}}, \overrightarrow{\mathrm{v}})}{\partial \overrightarrow{\mathrm{v}}}=-\frac{(1+d) \overrightarrow{\mathrm{vf}_{0}}(\overrightarrow{\mathrm{x}}, \overrightarrow{\mathrm{v}})}{1+\sum_{i} \mathrm{a}_{i} \mathrm{x}_{i}^{2}+\sum_{i} \mathrm{v}_{i}^{2}} .
$$

For an infinite plasma [6], the Cauchy distribution is very useful, since with it the formulas are much simpler and the equation was even exactly solvable in the 1D case; in higher dimensional cases, computations were much faster than for the normal. Using this distribution for the confined plasma does not offer any advantages; thus, we focus on a realistic normal distribution.

\section{F. The Green's functions}

In this subsection, we write the well-known expressions for the Poisson's equation Green's functions in the 1D, 2D and 3D spaces and their gradients. Using the introduced dimensionless units, we have

$$
\mathrm{G}\left(\overrightarrow{\mathrm{x}}, \overrightarrow{\mathrm{x}}^{\prime}\right)=-\frac{1}{2}\left|\overrightarrow{\mathrm{x}}-\overrightarrow{\mathrm{x}}^{\prime}\right|, \quad \frac{\partial}{\partial \overrightarrow{\mathrm{x}}} \mathrm{G}\left(\overrightarrow{\mathrm{x}}, \overrightarrow{\mathrm{x}}^{\prime}\right)=-\frac{1}{2} \operatorname{sign}\left(\overrightarrow{\mathrm{x}}-\overrightarrow{\mathrm{x}}^{\prime}\right),
$$

$$
\mathrm{G}\left(\overrightarrow{\mathrm{x}}, \overrightarrow{\mathrm{x}}^{\prime}\right)=-\frac{1}{2 \pi} \ln \left|\overrightarrow{\mathrm{x}}-\overrightarrow{\mathbf{x}}^{\prime}\right|, \quad \frac{\partial}{\partial \overrightarrow{\mathrm{x}}} \mathrm{G}\left(\overrightarrow{\mathrm{x}}, \overrightarrow{\mathbf{x}}^{\prime}\right)=-\frac{1}{2 \pi} \frac{\overrightarrow{\mathbf{x}}-\overrightarrow{\mathbf{x}}^{\prime}}{\left|\overrightarrow{\mathbf{x}}-\overrightarrow{\mathbf{x}}^{\prime}\right|^{2}}
$$


$\mathrm{G}\left(\overrightarrow{\mathrm{x}}, \overrightarrow{\mathbf{x}}^{\prime}\right)=\frac{1}{4 \pi} \frac{1}{\left|\overrightarrow{\mathrm{x}}-\overrightarrow{\mathbf{x}}^{\prime}\right|}, \quad \frac{\partial}{\partial \overrightarrow{\mathbf{x}}} \mathrm{G}\left(\overrightarrow{\mathbf{x}}, \overrightarrow{\mathbf{x}}^{\prime}\right)=-\frac{1}{4 \pi} \frac{\overrightarrow{\mathbf{x}}-\overrightarrow{\mathbf{x}}^{\prime}}{\left|\overrightarrow{\mathbf{x}}-\overrightarrow{\mathbf{x}}^{\prime}\right|^{3}}$,

for the $1 \mathrm{D}, 2 \mathrm{D}$, and $3 \mathrm{D}$ spaces, respectively.

\section{G. Dimensionless units for the integral equation}

Using the dimensionless units, we rewrite the integral equation (48) as follows:

$$
\mathrm{F}(\overrightarrow{\mathrm{x}}, \mathbf{s})=\mathrm{N}_{1}(\overrightarrow{\mathrm{x}}, \mathbf{s})-\int \mathrm{N}_{1}\left(\overrightarrow{\mathrm{x}}^{\prime}, \mathrm{s}\right) \mathrm{K}\left(\overrightarrow{\mathrm{x}}, \overrightarrow{\mathrm{x}}^{\prime}, \mathrm{s}\right) d \overrightarrow{\mathrm{x}}^{\prime} .
$$

To simplify the expressions, we introduce the following notation:

$$
\mathrm{R}\left(\overrightarrow{\mathrm{x}}, \overrightarrow{\mathbf{x}}^{\prime}, \overrightarrow{\mathrm{v}}\right)=F_{\mathrm{d}} \frac{\partial \mathrm{G}\left(\overrightarrow{\mathrm{x}}, \overrightarrow{\mathbf{x}}^{\prime}\right)}{\partial \overrightarrow{\mathrm{x}}} \frac{\partial \mathrm{f}_{0}(\overrightarrow{\mathbf{x}}, \overrightarrow{\mathrm{v}})}{\partial \overrightarrow{\mathrm{v}}},
$$

where $F_{\mathrm{d}}$ is a dimensionless constant, $F_{\mathrm{d}}=f_{d} v_{\mathrm{rms}}^{d} r_{\mathrm{D}}^{d}$; the derivatives in this expression are given by equations (79)-(81), (70), and (78). For the method for general orbits, we have

$\mathrm{K}\left(\overrightarrow{\mathbf{x}}, \overrightarrow{\mathbf{x}}^{\prime}, \mathrm{s}\right)=\int_{0}^{\infty} \int \mathrm{R}\left(\overrightarrow{\mathrm{X}}_{0}(0), \overrightarrow{\mathbf{x}}^{\prime}, \overrightarrow{\mathrm{V}}_{0}(0)\right) d \overrightarrow{\mathrm{v}} \mathrm{e}^{-\mathrm{st}} d \mathrm{t}$,

$\mathrm{F}(\overrightarrow{\mathrm{x}}, \mathrm{s})=-Z \iint_{0}^{\infty} \int_{0}^{\mathrm{t}} \mathrm{R}\left(\overrightarrow{\mathrm{X}}_{0}\left(\mathrm{t}_{1}\right), \overrightarrow{\mathrm{Y}}\left(\mathrm{t}_{1}\right), \overrightarrow{\mathrm{V}}_{0}\left(\mathrm{t}_{1}\right)\right) \mathrm{e}^{-\mathrm{st}} d \mathrm{t}_{1} d \mathrm{t} d \overrightarrow{\mathrm{v}}$,

and, for the method for periodic orbits,

$\mathrm{K}_{\mathrm{t}}\left(\overrightarrow{\mathrm{x}}, \overrightarrow{\mathrm{x}}^{\prime}, \mathrm{s}\right)=\frac{\mathrm{e}^{-\mathrm{ts}}}{\mathrm{e}^{\frac{2 \pi}{\bar{\omega}} \mathrm{s}}-1} \int_{t}^{\mathrm{t}+\frac{2 \pi}{\omega}} \int \mathrm{R}\left(\overrightarrow{\mathrm{X}}_{0}\left(\mathrm{t}_{1}\right), \overrightarrow{\mathrm{x}}^{\prime}, \overrightarrow{\mathrm{V}}_{0}\left(\mathrm{t}_{1}\right)\right) \mathrm{e}^{\mathrm{st}} d \overrightarrow{\mathrm{v}} d \mathrm{t}_{1}$,

$$
\begin{aligned}
\mathrm{F}_{\mathrm{t}}(\overrightarrow{\mathrm{X}}, \mathrm{s})= & -Z \frac{\mathrm{e}^{-\mathrm{ts}}}{\mathrm{e}^{\frac{2 \pi}{\tilde{\omega}} \mathrm{s}}-1} \int_{0}^{\infty} \int_{\mathrm{t}}^{\mathrm{t}+\frac{2 \pi}{\omega}} \int \mathrm{R}\left(\overrightarrow{\mathrm{X}}_{0}\left(\mathrm{t}_{1}\right), \overrightarrow{\mathrm{Y}}\left(\mathrm{t}_{1}\right), \overrightarrow{\mathrm{V}}_{0}\left(\mathrm{t}_{1}\right)\right) \\
& \times \mathrm{e}^{\left(\mathrm{t}_{1}-\mathrm{t}_{2}\right) \mathrm{s}} d \overrightarrow{\mathrm{v}} d \mathrm{t}_{1} d \mathrm{t}_{2},
\end{aligned}
$$

where $t$, as mentioned, is a free constant that can be set to zero.

\section{NUMERICAL METHODS}

In the previous section, we derived the LaplaceFredholm integral equation for the test charge problem for the confined plasma. In this section, we describe a numerical method to solve such equation. It has two main steps: solving the Fredholm integral equation for the Laplace image of the unknown function and then doing the inverse Laplace transform. We detail these steps in order.

\section{A. Numerical solution of the Fredholm equation}

Numerical methods for the Fredholm equations are very well developed [14], however, there are no publicly available codes implementing them. In this subsection, we review the piecewise polynomial collocation method (PPCM), emphasizing implementation details; for the mathematical details, such as the existence theorems and accuracy estimates, we refer to [14]. The idea of the method is to split the equation's domain, which should be a $d$-dimensional rectangle, into subdomains and then to represent the solution in each subdomain as polynomial series. The polynomials should be of some special form to transform the integral equation into a system of linear equations for the coefficients of these expansions, when the polynomial solution is inserted into the equation and evaluated at some special points.

Let us consider an integral equation

$$
\mathrm{F}(\overrightarrow{\mathbf{x}})=\mathrm{N}_{1}(\overrightarrow{\mathbf{x}})-\int_{G} \mathrm{~N}_{1}\left(\overrightarrow{\mathbf{x}}^{\prime}\right) \mathrm{K}\left(\overrightarrow{\mathbf{x}}, \overrightarrow{\mathbf{x}}^{\prime}\right) d \overrightarrow{\mathbf{x}}^{\prime},
$$

defined on a $d$-dimensional rectangular domain

$$
G=\prod_{i=1}^{d}\left[0, \mathrm{~b}_{i}\right]
$$

In every dimension $k$, we introduce $2 N_{k}+1$ grid points:

$$
\begin{gathered}
\mathrm{x}_{k}^{j}=\frac{\mathrm{b}_{k}}{2}\left(\frac{j}{N_{k}}\right)^{r}, \quad j=0, \ldots, N_{k}, \\
\mathrm{x}_{k}^{N_{k}+j}=\mathrm{b}_{k}-\mathrm{x}_{k}^{N_{k}-j}, \quad j=1, \ldots, N_{k},
\end{gathered}
$$

where $r$ is a constant, $r \geq 1$, that characterizes uniformity of the grid, i.e., for $r>1$, the grid becomes denser towards the boundaries of the domain, and, for $r=1$, it is uniform. Using these grid points, we partition $G$ into the closed cells:

$G_{j_{1}, \ldots, j_{d}}=\left\{\overrightarrow{\mathbf{x}} \in \mathbb{R}^{d}: \mathbf{x}_{k}^{j_{k}} \leq \mathrm{x}_{k} \leq \mathrm{x}_{k}^{j_{k}+1}, k=1, \ldots, d\right\}$,

for all $j_{k}=0, \ldots, 2 N_{k}$ for all $k=1, \ldots, d$. We then introduce $d$-dimensional multi-index $\mathbf{j}, \mathbf{j}=\left(j_{1}, \ldots, j_{d}\right)$, such that $G_{j_{1}, \ldots, j_{d}} \equiv G_{\mathbf{j}}$ and

$$
G=\underset{\mathbf{j}}{G_{\mathbf{j}}}
$$


Then we choose $m$ interpolation points in $[-1,1]:-1 \leq$ $\xi^{1}<\ldots<\xi^{m} \leq 1$ and map them into every dimension of all partitions, thereby obtaining collocation points in each cell $G_{\mathbf{j}}$. We denote the collocation points as $\boldsymbol{\xi}^{\mathbf{j}, \mathbf{l}}$; it is a $d$-dimensional vector with two multi-indexes, where $\mathbf{j}$ characterizes the cell the collocation point belongs to and components of $\mathbf{I}$ runs from 1 to $m$ characterizing the mapped interpolation point. In Fig. 1, we depict this construction for $d=2$.

Then, we introduce the interpolating polynomials of degree $(m-1)^{d}$ that has two multi-indexes:

$$
\boldsymbol{\phi}^{\mathbf{j}, \mathbf{m}}(\overrightarrow{\mathbf{x}})=\prod_{i=1}^{d} \prod_{q=1 \ldots m}^{q \neq m_{i}} \frac{\left(\mathbf{x}_{i}-\xi_{i}^{j_{i}, q}\right)}{\left(\xi_{i}^{j_{i}, m_{i}}-\xi_{i}^{j_{i}, q}\right)}, \quad \overrightarrow{\mathbf{x}} \in G_{\mathbf{j}}
$$

where the first multi-index characterizes the cell, to which the polynomial belongs, and the second, each component of which runs from 1 to $m$, characterizes the collocation point used to build the polynomial. For example, for $m=1$, the polynomials will be just constants. The polynomials satisfy the following important property:

$$
\boldsymbol{\phi}^{\mathbf{j}, \mathbf{m}}(\boldsymbol{\xi} \mathbf{j , l})=\delta_{\mathbf{m}, \mathbf{l}},
$$

where $\delta_{\mathbf{m}, \mathbf{l}}$ is the Kronecker delta, defined for the multiindexes via

$$
\delta_{\mathbf{m}, \mathbf{l}}=\prod_{i=1}^{d} \delta_{m_{i}, l_{i}} .
$$

We look for a solution in the following form:

$$
\mathbf{N}_{1}(\overrightarrow{\mathbf{x}})=\sum_{\mathbf{m}} c_{\mathbf{j}}^{\mathbf{m}} \boldsymbol{\phi}^{\mathbf{j}, \mathbf{m}}(\overrightarrow{\mathbf{x}}), s \quad \overrightarrow{\mathbf{x}} \in G_{\mathbf{j}}
$$



FIG. 1. Cells and collocation points for the PPCM for $d=2$, $N_{1}=N_{2}=3, m=3, r=1$. The collocation points are shown only in three cells, while, of course, they exist in all of them. where the summation runs over all possible values of multiindex $\mathbf{m}$. We emphasize that unknown coefficients and polynomials are different in each cell $G_{\mathbf{j}}$.

Then we insert the solution (97) into the equation (88), and, using the condition that the equation is satisfied at the collocation points and property (95), we reduce the integral equation to the system of linear equations for $c_{\mathbf{j}}^{\mathbf{m}}$ :

$$
c_{\mathbf{i}}^{\mathbf{l}}=\sum_{\mathbf{j}} \sum_{\mathbf{m}} a_{\mathbf{i}, \mathbf{j}}^{\mathbf{l} \mathbf{m}} c_{\mathbf{j}}^{\mathbf{m}}+\mathbf{F}\left(\boldsymbol{\xi}^{\mathbf{i}, \mathbf{l}}\right),
$$

where

$$
a_{\mathbf{i}, \mathbf{j}}^{\mathbf{l}, \mathbf{m}}=\int_{G_{\mathbf{j}}} K\left(\boldsymbol{\xi}^{\mathbf{i}, \mathbf{l}}, \vec{y}\right) \boldsymbol{\phi}^{\mathbf{j}, \mathbf{m}}(\vec{y}) d \vec{y}
$$

and $F\left(\boldsymbol{\xi}^{\mathbf{i}, \mathbf{I}}\right)$ is just $\mathrm{F}(\overrightarrow{\mathbf{x}})$ evaluated at the collocation point $\xi^{\mathbf{i}, 1}$. Equations (98) ensure that the numerical solution (97) equals the unknown true solution at all collocation points; at other points, the numerical solution is interpolated by polynomials. Obviously, the matrix $a_{\mathbf{i}, \mathbf{j}}^{\mathbf{l}, \mathbf{m}}$, vector $\mathrm{F}\left(\boldsymbol{\xi}^{\mathbf{i}, \mathbf{l}}\right)$ and the unknown vector of coefficients $c_{\mathrm{i}}^{\mathrm{l}}$ can be represented as a matrix with two scalar indexes and two vectors with one scalar index, i.e., $\mathbf{A}, \vec{f}$, and $\vec{c}$, respectively, and standard methods to solve systems of linear equations can be applied. With these notations, the system (98) looks as follows:

$$
(\mathbf{I}-\mathbf{A}) \vec{c}=\vec{f}
$$

where $\mathbf{I}$ is an identity matrix. Dimension of matrix $\mathbf{A}$ is $\prod_{k=1}^{d}\left(2 N_{k} m_{k}\right)$.

It is worth it to note that this method can deal with integral equations with weakly singular kernels, meaning that the kernels can have singularities, but they must be integrable [14].

We developed our own multidimensional integrators to compute integrals in (99). Multidimensional integral can be computed recursively using one-dimensional integrators. At each step of recursion, we use as an integrand the initial integral corresponding to this step, but with lowered by one number of integration variables. We also store the values of this step's integration variables and pass them to further steps. When dimension of the integral is one, the recursive integrator takes function as an integrand and evaluates it at the values of the integration variables, which were stored in previous steps of the recursion. To perform onedimensional integration, we implemented the adaptive Gauss-Kronrod algorithm. We also used this integrator to evaluate integrals in expressions for the kernel and the left-hand side of the integral equations corresponding to the test charge problem. 


\section{B. Numerical solution of the Laplace-Fredholm equation}

The integral equation for the test charge problem (82) is slightly more complicated than the Fredholm equation (88), i.e., we have the Fredholm equation

$$
\mathrm{F}(\overrightarrow{\mathbf{x}}, \mathbf{s})=\mathrm{N}_{1}(\overrightarrow{\mathbf{x}}, \mathbf{s})-\int \mathrm{N}_{1}\left(\overrightarrow{\mathbf{x}}^{\prime}, \mathbf{s}\right) \mathrm{K}\left(\overrightarrow{\mathbf{x}}, \overrightarrow{\mathbf{x}}^{\prime}, \mathbf{s}\right) d \overrightarrow{\mathbf{x}}^{\prime},
$$

but for the Laplace image of the quantity of interest. It is well known that the inverse Laplace transform of a known function $\tilde{f}(s)$ can be computed using discrete Fourier transform (A12):

$f\left(t_{j}\right)=\mathrm{e}^{\sigma t_{j}} C_{j} \mathrm{DFT}_{j}\left[\left\{\tilde{f}\left(\sigma+i k_{n}\right)(-1)^{n}\right\}_{0 \leq n \leq N-1}\right]$,

this formula is derived and all notations are explained in Appendix A; for more details, we refer to [6], where numerical tests were also performed. The discrete Fourier transform can be computed numerically using the fast Fourier transform (FFT) algorithm. For any value of $\mathrm{s}$, we can obtain the numerical solution of the integral equation (101) via the PPCM described in previous subsection. To invert the Laplace transform numerically, we need to solve the equation (101) for $\mathrm{s}=\sigma+i \mathrm{k}_{n}$ for some set $\left\{\mathrm{k}_{n}\right\}_{0 \leq n \leq N-1}$. Then, we store these solutions for d-dimensional array of $\vec{x}$ values of interest; thereafter, for each value of $\vec{x}$, we invert the Laplace transform via (102). After solving equations (101) for the set of $S$, we have the following $(d+1)$-dimensional array of solutions:

$$
\left\{\mathrm{N}_{1}\left(\mathbf{x}_{i_{1}}, \ldots, \mathbf{X}_{i_{d}}, \sigma+i \mathrm{k}_{n}\right)\right\}_{0 \leq n \leq N-1,0 \leq i_{j} \leq N_{j}^{(x)}, 0 \leq j \leq d-1},
$$

where $N_{j}^{(x)}$ is the number of grid points in dimension $j$. Inverting the Laplace transform via (102), we obtain the space-time solution:

$$
\begin{aligned}
& \mathrm{n}_{1}\left(\overrightarrow{\mathrm{x}}_{\mathbf{i}}, t_{j}\right) \\
& \quad=\mathrm{e}^{\sigma t_{j}} C_{j} \times \mathrm{DFT}_{j}\left[\left\{\mathrm{~N}_{1}\left(\overrightarrow{\mathrm{x}}_{\mathbf{i}}, \sigma+i \mathrm{k}_{n}\right)(-1)^{n}\right\}_{0 \leq n \leq N-1}\right],
\end{aligned}
$$

where $\overrightarrow{\mathbf{x}}_{\mathbf{i}}=\left(\mathbf{x}_{i_{1}}, \ldots, \mathbf{x}_{i_{d}}\right)$. The discrete Fourier transform in this formula can be evaluated via the FFT; the numerical test of this procedure is discussed in Sec. V.

Thus, being able to solve Eq. (101) over some grid of complex numbers for $\mathrm{S}$, we can obtain the space-time density $\mathrm{n}_{1}(\overrightarrow{\mathrm{x}}, t)$ via (104). For complex values of $\mathrm{s}$, Eq. (101) turns into a system of equations for $\operatorname{ReN}_{1}\left(\overrightarrow{\mathbf{x}}^{\prime}, \mathbf{s}\right)$ and $\operatorname{ImN}_{1}\left(\overrightarrow{\mathbf{x}}^{\prime}, \mathbf{s}\right)$ :
$\left(\begin{array}{c}\operatorname{ReF} \\ \operatorname{ImF}\end{array}\right)=\left(\begin{array}{c}\operatorname{ReN}_{1} \\ \operatorname{ImN}_{1}\end{array}\right)-\int_{G}\left(\begin{array}{cc}\operatorname{ReK} & -\operatorname{ImK} \\ \operatorname{ImK} & \operatorname{ReK}\end{array}\right)\left(\begin{array}{c}\operatorname{ReN}_{1} \\ \operatorname{ImN}_{1}\end{array}\right) d \overrightarrow{\mathbf{x}}^{\prime}$

wherein, for brevity, we omitted arguments of functions. The PPCM can be applied to this system as well. Looking for solution in a form similar to (97),

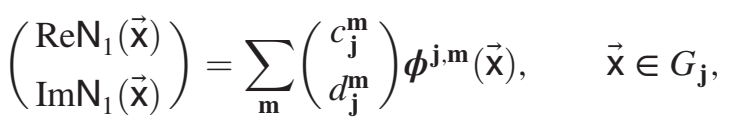

we obtain a system of linear equations analogous to (100):

$$
\left(\begin{array}{cc}
\mathbf{I}-\mathbf{A} & \mathbf{B} \\
-\mathbf{B} & \mathbf{I}-\mathbf{A}
\end{array}\right)\left(\begin{array}{l}
\vec{c} \\
\vec{d}
\end{array}\right)=\left(\begin{array}{l}
\vec{f} \\
\vec{g}
\end{array}\right)
$$

where $\mathbf{A}$ and $\vec{f}$ have the same meaning as in (100), but for the real parts of the corresponding quantities, and $\mathbf{B}$ and $\vec{g}$-for the imaginary parts; vectors $\vec{c}$ and $\vec{d}$ are the unknown vectors corresponding to solution (106). The dimension of the matrix of the system (107) is $\prod_{k=1}^{d}\left(4 N_{k} m_{k}\right)$.

\section{Implementation and tests}

The algorithm described in the previous section we implemented as a parallel object-oriented program. There are two natural ways to parallelize the algorithm. To do the Laplace transform in (104), we need to have the solutions of the integral equation (101) for different values of the parameter $\mathrm{S}$ that are independent and can be obtained in parallel. The most time-consuming part of solving the individual integral equation is the evaluation of the matrix elements of $\mathbf{A}$, or, in complex case, of the matrix of the system of linear equations (107). Each matrix element is a multidimensional integral (99) and they are also independent of each other; thus, they can be evaluated in parallel. Our solver deals with abstract classes for the kernel and for the left-hand side of an integral equation. By defining subclasses of these classes and supplying them to the solver, we can solve equations corresponding to different problems.

It is easy to construct an exactly solvable integral equation by taking some functions for the solution and for the kernel, and then, the left-hand side can be obtained via substituting these functions into the equation. For example, a $d$-dimensional equation

$\mathrm{F}(\overrightarrow{\mathbf{x}}, \mathbf{s})=\mathrm{N}(\overrightarrow{\mathbf{x}}, \mathbf{s})-\int_{[-\pi, \pi]^{d}} \mathrm{~N}\left(\overrightarrow{\mathbf{x}}^{\prime}, \mathbf{s}\right) \mathrm{K}\left(\overrightarrow{\mathbf{x}}, \overrightarrow{\mathbf{x}}^{\prime}, \mathbf{s}\right) d \overrightarrow{\mathbf{x}}^{\prime}$

with a kernel 


$$
\mathrm{K}\left(\overrightarrow{\mathbf{x}}, \overrightarrow{\mathbf{x}}^{\prime}, \mathbf{s}\right)=\mathrm{s}^{2} \prod_{i=1}^{d} \cos \mathbf{x}_{i}^{\prime} \sin \mathbf{x}_{i}
$$

and a left-hand side

$$
\mathrm{F}(\overrightarrow{\mathbf{x}}, \mathbf{s})=\frac{1}{\mathbf{s}^{2}} \prod_{i=1}^{d} \sin \mathbf{x}_{i}
$$

has an exact solution

$$
\mathrm{N}(\overrightarrow{\mathbf{x}}, \mathbf{s})=\frac{1}{\mathbf{s}^{2}} \prod_{i=1}^{d} \sin \mathbf{x}_{i}
$$

and in the time domain:

$$
\mathrm{n}(\overrightarrow{\mathrm{x}}, \mathrm{t})=\mathrm{t} \prod_{i=1}^{d} \sin \mathrm{x}_{i}, \quad \mathrm{t} \geq 0
$$

In Fig. 2, we show the numerical solution in the time domain of this equation obtained by our program for $d=1$. It is indistinguishable from the exact solution (112), the extrema of the curves equal the corresponding time values, as they should accordingly to (112). We also completed the same tests for higher dimensions.

The solver is implemented in C++ using MPI for parallelization. The results shown in Fig. 2 were obtained on Hopper machine at the National Energy Research Scientific Computing Center (NERSC); Hopper is a Cray XE6 supercomputer with 6,384 nodes, which are two twelve-core AMD "Magny-Cours" $2.1 \mathrm{GHz}$ processors. We utilized 256 nodes (6,144 cores), it took $2.52 \mathrm{~s}$ to solve this exactly solvable Laplace-Fredholm equation; individual Fredholm equations (for a given s) were solved in $0.02 \mathrm{~s}$.

The complete source code of our program is distributed under the terms of the GNU General Public License at [15]. The code includes a stand-alone solver for the

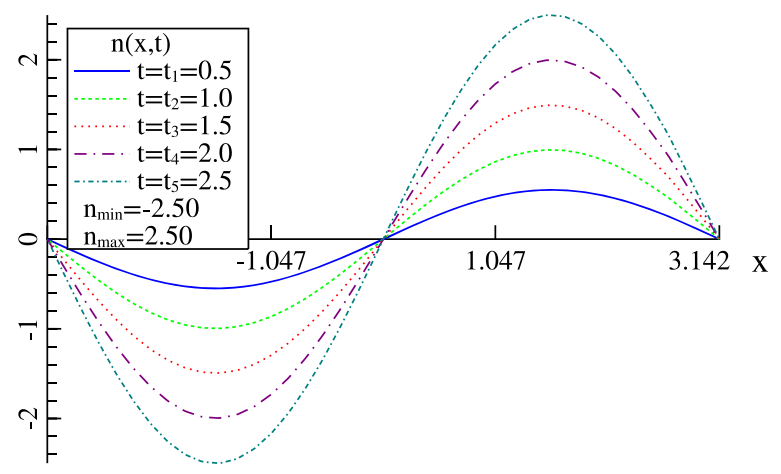

FIG. 2. Numerical solution of the 1D Laplace-Fredholm equation (108) with kernel (109) and left-hand side (110) obtained via the PPCM and Laplace transform inversion via FFT. multidimensional Fredholm integral equations of the second type, a solver for the Laplace-Fredholm equations and the kernel and left-hand side classes for the test charge problem. The codes for all particular cases considered in Sec. VI are included.

\section{TEST CHARGE PROBLEM FOR AN INFINITE PLASMA}

In this section, we discuss some of our results for an infinite plasma; for more details, we refer to [6]. As we already mentioned, in this case, the Vlasov-Poisson system is the same as for the confined plasma that we consider in the present article, but, in the Vlasov equation (18), the third term equals zero. This makes the system solvable via the Laplace and Fourier transforms. The electron density perturbation, resulting from the interaction of an infinite plasma with an external charge moving along a straight line $\vec{y}(t)=\vec{x}_{0}+\vec{v}_{0} t$, and valid in the 1D, 2D, and 3D spaces, is given by [6]:

$\mathrm{n}_{1}(\overrightarrow{\mathbf{x}}, \mathrm{t})=\mathrm{L}^{-1} \mathrm{~F}^{-1}\left[\frac{\mathrm{e}^{-i \overrightarrow{\mathrm{k}} \cdot \overrightarrow{\mathrm{x}}_{0}}}{\left(\frac{f_{d}^{-1} v_{\mathrm{rms}}^{-d}}{\mathrm{LF}_{\overrightarrow{\mathrm{k}}_{\mathrm{kt}}}\left(\mathrm{t}_{0}(\overrightarrow{\mathrm{v}})\right)}+1\right)\left(\mathrm{s}+i \overrightarrow{\mathrm{k}} \cdot \overrightarrow{\mathrm{v}}_{0}\right)}\right]$,

where

$$
\operatorname{LF}_{\vec{k} t}\left(\mathrm{tf}_{0}(\overrightarrow{\mathrm{v}})\right)=\int_{0}^{\infty} \mathrm{e}^{-\mathrm{ts} t} \int \mathrm{f}_{0}(\overrightarrow{\mathrm{v}}) \mathrm{e}^{-i \vec{k} \cdot \overrightarrow{\mathrm{v}} \mathrm{t}} d \overrightarrow{\mathrm{v}} d \mathrm{t}
$$

$f_{d}$ and $\mathrm{f}_{0}(\overrightarrow{\mathrm{v}})$ are defined in (56), $f_{d}^{-1} v_{\mathrm{rms}}^{-d}$ is a dimensionless factor, and $\mathrm{L}^{-1}$ and $\mathrm{F}^{-1}$ are the inverse Laplace and Fourier transform operators for the dimensionless variables, respectively.

We consider this solution for two particular equilibrium densities $\mathrm{f}_{0}(\overrightarrow{\mathrm{v}})$; for each case, we only need to compute $\mathrm{LF}_{\overrightarrow{\mathrm{kt}}}\left(\mathrm{tf}_{0}(\overrightarrow{\mathrm{v}})\right)$ and $f_{d}^{-1} v_{\text {rms }}^{-d}$, and then insert them into (113). For the 1D Cauchy distribution

$$
\mathrm{f}_{0}(\overrightarrow{\mathrm{v}})=\frac{1}{\pi\left(1+\mathrm{v}^{2}\right)},
$$

the inverse integral transforms in (113) can be evaluated analytically giving the following expression [6]:

$$
\begin{aligned}
\mathrm{n}_{1}(\overrightarrow{\mathrm{x}}, \mathrm{t})= & \frac{1}{4 \pi} \frac{1}{\mathrm{v}_{0}-i}\left(\mathrm{e}^{-\mathcal{A}_{+}}\left(\operatorname{Ei}\left(\mathcal{A}_{+}\right)-\operatorname{Ei}\left(\mathcal{B}_{+}\right)\right)\right. \\
& \left.+\mathrm{e}^{\mathcal{A}_{+}}\left(\mathrm{E}_{1}\left(\mathcal{A}_{+}\right)-\mathrm{E}_{1}\left(\mathcal{B}_{+}\right)\right)\right) \\
& +\frac{1}{4 \pi} \frac{1}{\mathrm{v}_{0}+i}\left(\mathrm{e}^{-\mathcal{A}_{-}}\left(\operatorname{Ei}\left(\mathcal{A}_{-}\right)-\operatorname{Ei}\left(\mathcal{B}_{-}\right)\right)\right. \\
& \left.+\mathrm{e}^{\mathcal{A}_{-}}\left(\mathrm{E}_{1}\left(\mathcal{A}_{-}\right)-\mathrm{E}_{1}\left(\mathcal{B}_{-}\right)\right)\right),
\end{aligned}
$$

where 


$$
\mathcal{A}_{ \pm}=\frac{\mathrm{tv}_{0}-\mathrm{x}+\mathrm{x}_{0}}{1 \pm i \mathrm{v}_{0}}, \quad \mathcal{B}_{ \pm}=\frac{\mathrm{x}_{0}-\mathrm{x} \pm i \mathrm{t}}{1 \pm i \mathrm{v}_{0}}
$$

and $\mathrm{E}_{1}(z)$ and $\operatorname{Ei}(z)$ are the exponential integral functions [13] that can be computed via the series expansions [6]. Computations show that the expression (116) is real even though it contains complex numbers.

For other distributions and, in particular, for the 1D normal distribution

$$
\mathrm{f}_{0}(\overrightarrow{\mathrm{v}})=\pi^{-\frac{d}{2}} \mathrm{e}^{-\mathrm{v}^{2}}
$$

we were unable to derive an analytical expression for the solution, and so used the FFT algorithm to evaluate the inverse integral transforms in (113) numerically.

Figure 3 compares the density perturbation for the 1D Cauchy distribution computed using the analytical expression (116) and the density obtained numerically via (113) using the FFT techniques that we detail in Appendix A. This plot shows that values obtained numerically via the FFT agree perfectly with the exact values. We use the same numerical methods to invert the integral transforms in (104) in our method for the Laplace-Fredholm equation.

In Fig. 4, we show plots for the density obtained via the expression (113) numerically for the 1D Cauchy and normal distributions. For some time values, we see negative and smaller additional positive peaks. The main positive peaks follow the external charge with some delay.

In Fig. 3, for the density perturbation for the 1D Cauchy distribution, for $t=10.61$, we see a negative peak behind the main positive peak and another smaller positive peak behind the negative one; for $t=12.54$, we see a larger negative peak. Negative and small positive peaks can also be seen in Fig. 4 for the normal distribution for $t=5.0$. These are standard plasma oscillations, which are caused by appearance of the external charge in plasma.

An initial wavelike disturbance in plasma results in a damped traveling wave-this is well-known Landau damping [16]. Any disturbance can be represented as a

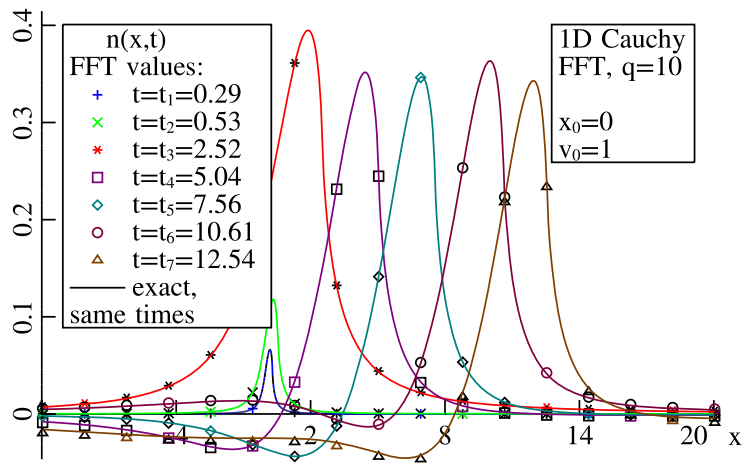

FIG. 3. The density $n_{1}(\vec{x}, t)$ for the infinite plasma with the 1D Cauchy velocity distribution obtained via exact formula (116) and numerically via the FFT.
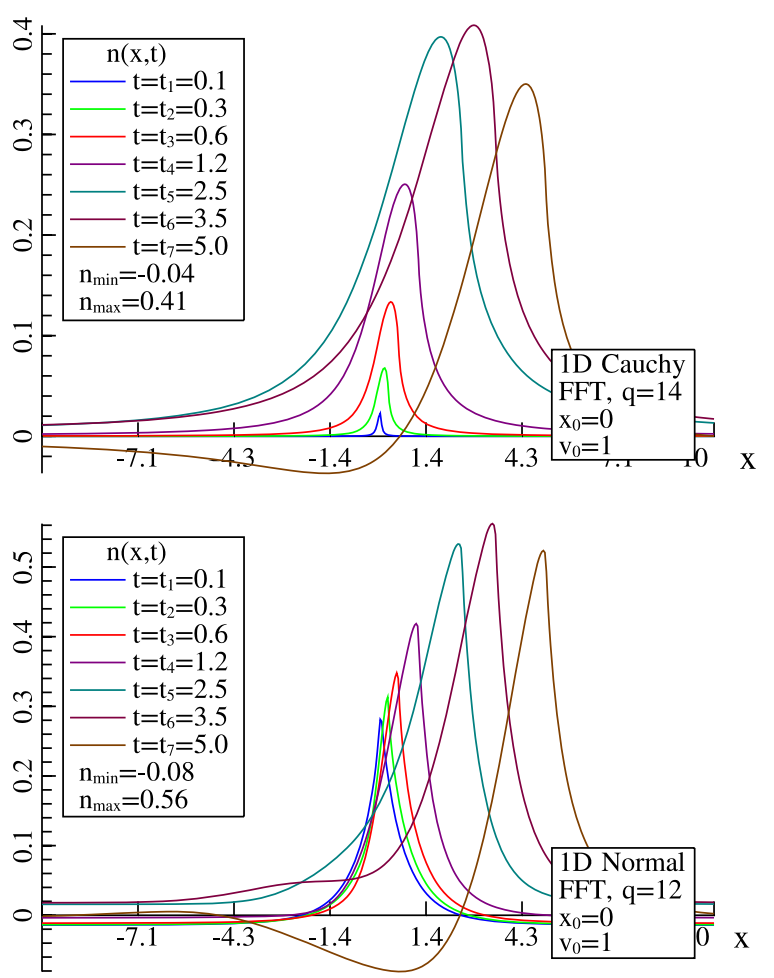

FIG. 4. The density $n_{1}(\vec{x}, t)$ for the infinite plasma with the $1 D$ Cauchy and normal velocity distributions.

superposition of such disturbances via Fourier integral, that means that Landau damping should be present in the solution of the test charge problem as well. However, unlike in classical Landau's consideration, in our case, the external excitation (charge) does not disappear immediately, but continues to exist in plasma. As a result, the Landau damping will lead to decay of the oscillations of the maximum of the perturbation, as we see in Fig. 3, and stationary perturbation will be established at infinity, as it was shown in [5].

The numerical results for other $1 \mathrm{D}$ distributions and $2 \mathrm{D}$ and 3D plasmas can be found in [6]; they have the same qualitative features.

In Sec. VI, we shall see the results for the confined plasma, which also have these qualitative features.

\section{NUMERICAL RESULTS}

In Sec. III, we derived two different integral equations for the test charge problem in a confined plasma. One equation can deal with both confined and infinite plasmas; the kernel and the left-hand side are given by the formulas (84) and (85), respectively. Although, this method can deal with infinite plasmas, the numerical method that we described in Sec. IV is only for compact domains; some modifications are required to solve the equations with infinite domains, which we are not going to discuss here. For infinite plasmas, there are much simpler methods that are thoroughly developed in [6]. The second integral 
equation with the kernel and the left-hand side given by (86) and (87) can deal only with a spherical confined plasma; however, it has simpler integrals over time that can be computed faster. We did numerical tests of both methods for a confined plasma and obtained the same results, this confirms that the methods are equivalent for spherical plasmas, as they should be according to our theory.

In this section, we focus on a confined spherical plasma with the normal (Maxwell) velocity and spatial distributions given by (69). The components of vector $\vec{a}$ in the expression for the equilibrium distribution characterize the plasma's size; for example, when they all equal one, $\sigma$ also equals one, and more than $99.9 \%$ of the plasma lie within a ball of radius 3, accordingly to considerations in Sec III E 1.

We will graphically present our results obtained via the second method. We show plots for some time values, but we would like to emphasize that, when solution is obtained, it is available for all time points in a certain predefined range. We consider the 1D, 2D, and 3D cases in order. Then we will discuss our results.

We also assume that perturbation is zero outside of the $d$-dimensional rectangle $\prod_{j=1}^{d}\left[-4 \sigma_{j}, 4 \sigma_{j}\right]$, where $\sigma_{j}$ is defined via (72), and take this rectangle as a domain of the integral equations. In all plots, the time and distance are measured in units of inverse plasma frequency and the Debye radius, defined in (53) and (54), respectively.

Since in the 1D case, the time dependence is quite simple, we depicted the densities with time step 0.5. In the 2D and 3D cases, it is more complicated; hence, we plotted it with a time step 0.1 and depicted for the values, for which important features of the dynamics of the density are more pronounced.

For simplicity, we did computations for the external charges moving with a constant velocity along straight lines; however, the method and our software can deal with charges moving along any trajectory.

All computations were performed on Edison machine at NERSC. Edison is a Cray XC30 supercomputer with 5,576 nodes, which are two twelve-core Intel "Ivy Bridge" $2.4 \mathrm{GHz}$ processors with $64 \mathrm{~GB}$ of RAM. The 1D case was computed on 2,048 cores in $14 \mathrm{~s}$. The $2 \mathrm{D}$ case was

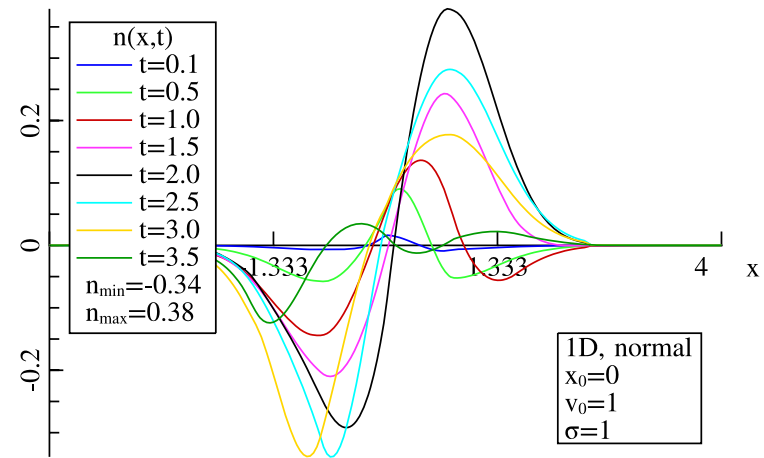

computed on 4,096 cores in 14 min and $12 \mathrm{~s}$. Computation of the 3D case was split into two steps: evaluation the matrix and right-hand side of the system (107), and solving this system via LU decomposition. The first step was done on 16,392 cores (16,392 MPI tasks, 683 nodes) in $2 \mathrm{~h}$, and the second step was done on 3,072 cores (256 MPI tasks, two tasks per node or one task per 12 cores-we needed more memory on each task) in $36 \mathrm{~min}$. Computation of the second step can be accelerated approximately by a factor of 10 by using parallel LU decomposition reducing computation time of the whole 3D problem to almost $2 \mathrm{~h}$. The great advantage of our program is its ability to utilize effectively thousands of cores available on modern supercomputers. Particle-in-cell simulation performed by VSim (formerly Vorpal) of the similar 3D test charge problem with interaction time about 4.5 plasma periods takes approximately $8 \mathrm{~h}$ on 12,288 cores (512 nodes) - a few times more than our computation.

\section{A. Numerical results for the 1D plasma}

In this case, we consider an external charge starting moving with velocity $v_{0}=1$ from the center of the plasma, $x_{0}=0$, and moving with the same velocity, but from outside of the plasma, i.e., $\mathrm{x}_{0}=-4$.

In Fig. 5, we show our results for the " $x_{0}=0$ " case and, in Fig. 6, for " $x_{0}=-4$."

A point charge in the 1D space corresponds to a charged plane perpendicular to $\mathrm{x}$ axis in a 3D plasma with the $\mathrm{X}$-dependent distribution, in our case, the normal distribution along $\mathrm{X}$ axis and the uniform with the infinite support (support of a function is a set of points where the function is nonzero) along two other axes. If this plane has equation $\mathrm{X}=\mathrm{C}$, where $\mathrm{C}$ is some constant, the potential and the charge density do not depend on $\mathrm{z}$ and $\mathrm{y}$ and equations are the same as we have in the 1D case.

\section{B. Numerical results for the 2D plasma}

In the $2 \mathrm{D}$ case, we consider an external charge starting moving from the center of the plasma along $\mathrm{X}$ axis with velocity $\vec{v}=(1.0,0.0)$.

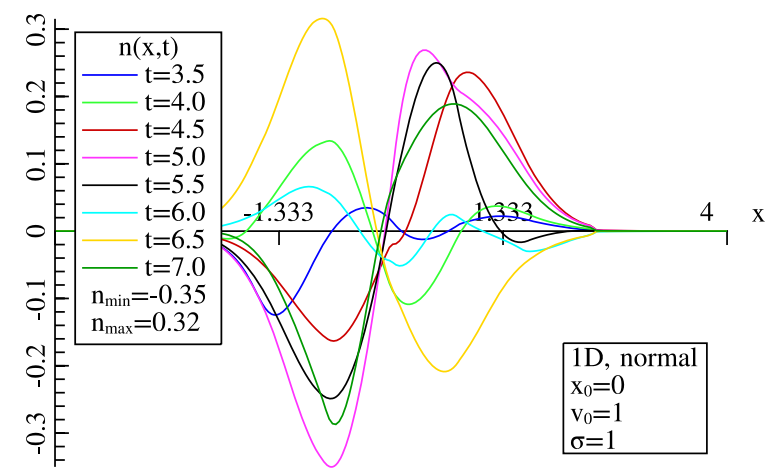

FIG. 5. Dynamics of the density $n(\overrightarrow{\mathbf{x}}, \mathrm{t})$ for the $1 \mathrm{D}$ plasma with the normal spatial and velocity distributions and for a charge starting moving from $x_{0}=0$. 

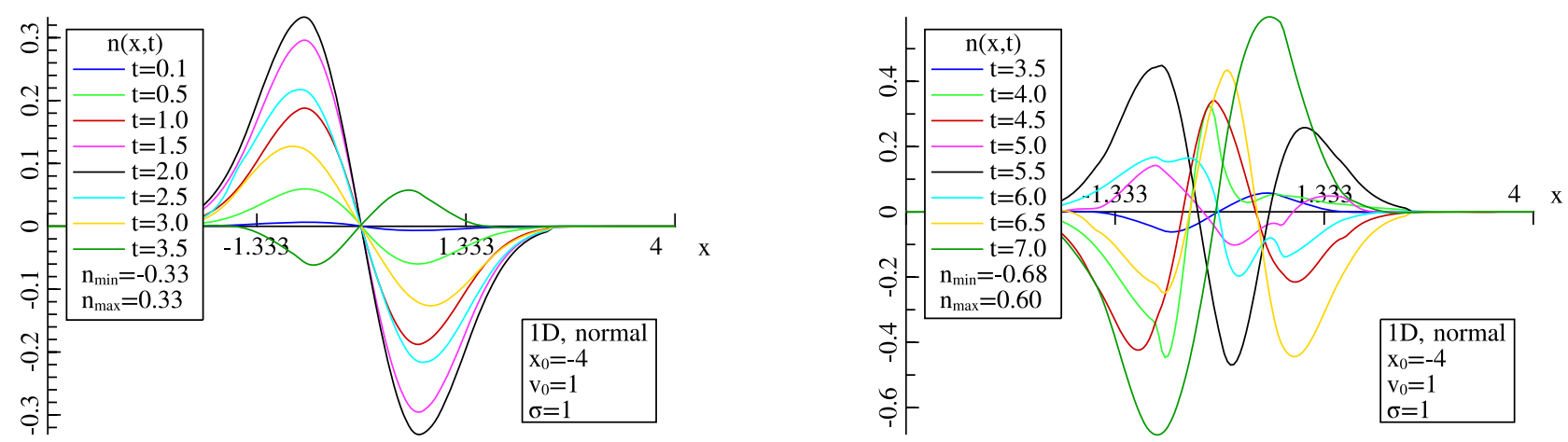

FIG. 6. Dynamics of the density $n(\overrightarrow{\mathrm{x}}, \mathrm{t})$ for the 1D plasma with the normal spatial and velocity distributions and for a charge starting moving from $\mathrm{x}_{0}=-4$.
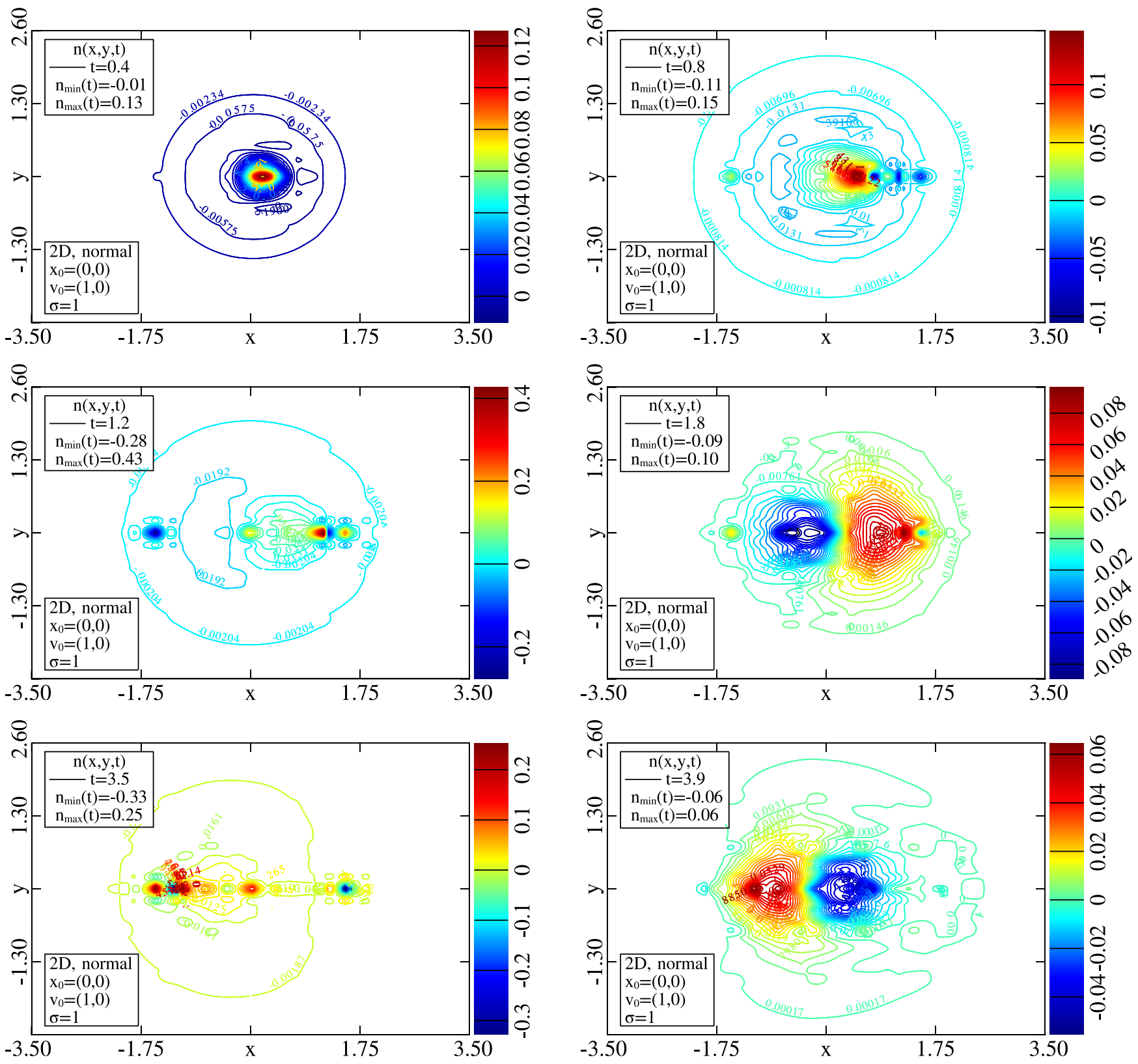

FIG. 7. Dynamics of the density $n(\overrightarrow{\mathrm{x}}, \mathrm{t})$ for the $2 \mathrm{D}$ plasma with normal spatial and velocity distributions for different values of time; the external charge starts moving from $\overrightarrow{\mathrm{x}}_{0}=(0.0,0.0)$ with velocity $\overrightarrow{\mathrm{v}}_{0}=(1.0,0.0)$; each plot has its own color bar. 
Figure 7 shows the electron density for different values of the time; these are the contour density plots, depicting lines, along which the density is constant; we call them the equidensity contours. For some contours, values of the density are shown on the graphs; for others, the values can be obtained via the color bars attached to the plots. As we see from the plots, for times smaller than one half, there is no significant negative peak, and the negative density is spread out around a positive peak, while for the 1D case, both peaks are of the same size from the beginning. For times greater than one, a negative peak appears behind the positive one; and they both become very spiky, then they widen for a time around two. Thereafter, the oscillations continue; for a time about 3.5 , the positive peak has negative $x$ coordinate and the negative-positive, and then they widen. These oscillations continue for larger times.

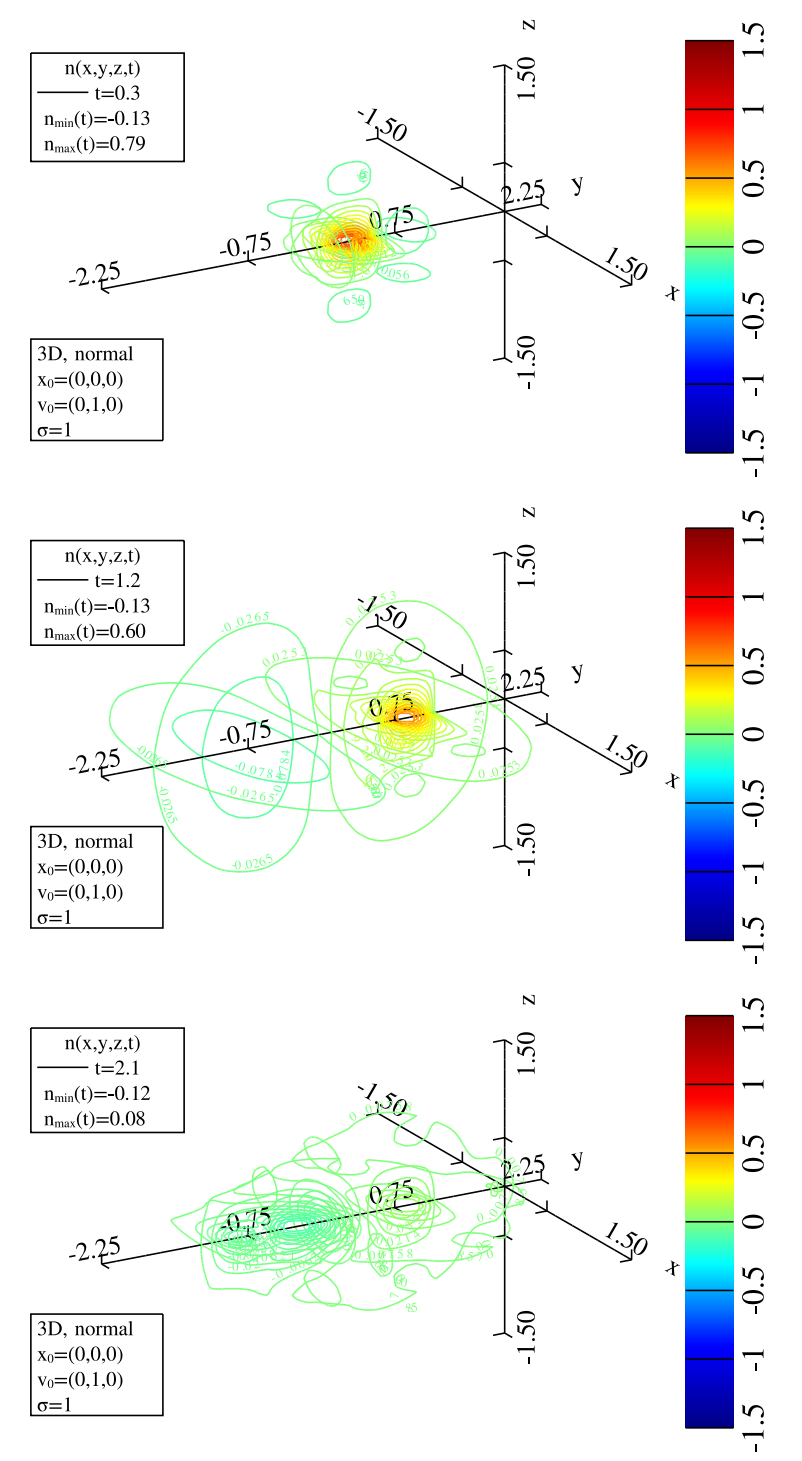

A point charge in the 2D space corresponds to a charged infinite line in a 3D plasma with the $\mathrm{z}$-independent distribution; in our case, it is an infinite cylinder with the normal distribution in transverse directions and uniform with infinite support in longitudinal.

\section{Numerical results for the 3D plasma}

In the 3D case, we consider the problem with an external charge starting moving from the center of the plasma along y axis with velocity $\vec{v}=(0.0,1.0,0.0)$. In this case, we show the results as 3D density plots, which depict equidensity contours in the planes $\mathbf{x}=0$ and $\mathbf{z}=0$; some plots also have few lines in the plane $y=0$.

Figures 8 and 9 depict the densities for different times. The dynamics is quite similar to that in the $2 \mathrm{D}$ case. In the beginning, there is a positive peak, which follows the

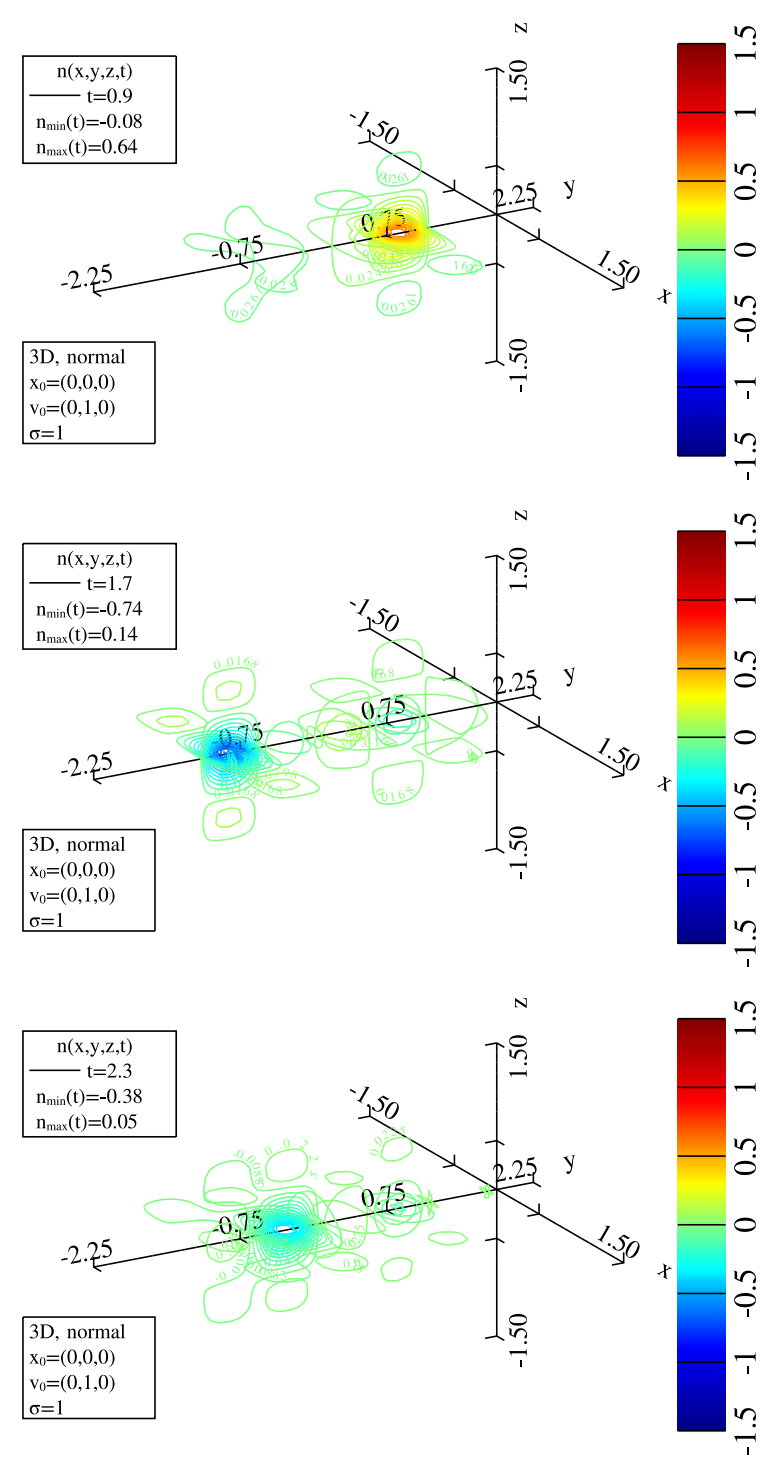

FIG. 8. Dynamics of the density $n(\overrightarrow{\mathrm{x}}, \mathrm{t})$ for the 3D plasma with normal spatial and velocity distributions for different values of time; the external charge starts moving from $\vec{x}_{0}=(0.0,0.0,0.0)$ with velocity $\vec{v}_{0}=(0.0,1.0,0.0)$. 

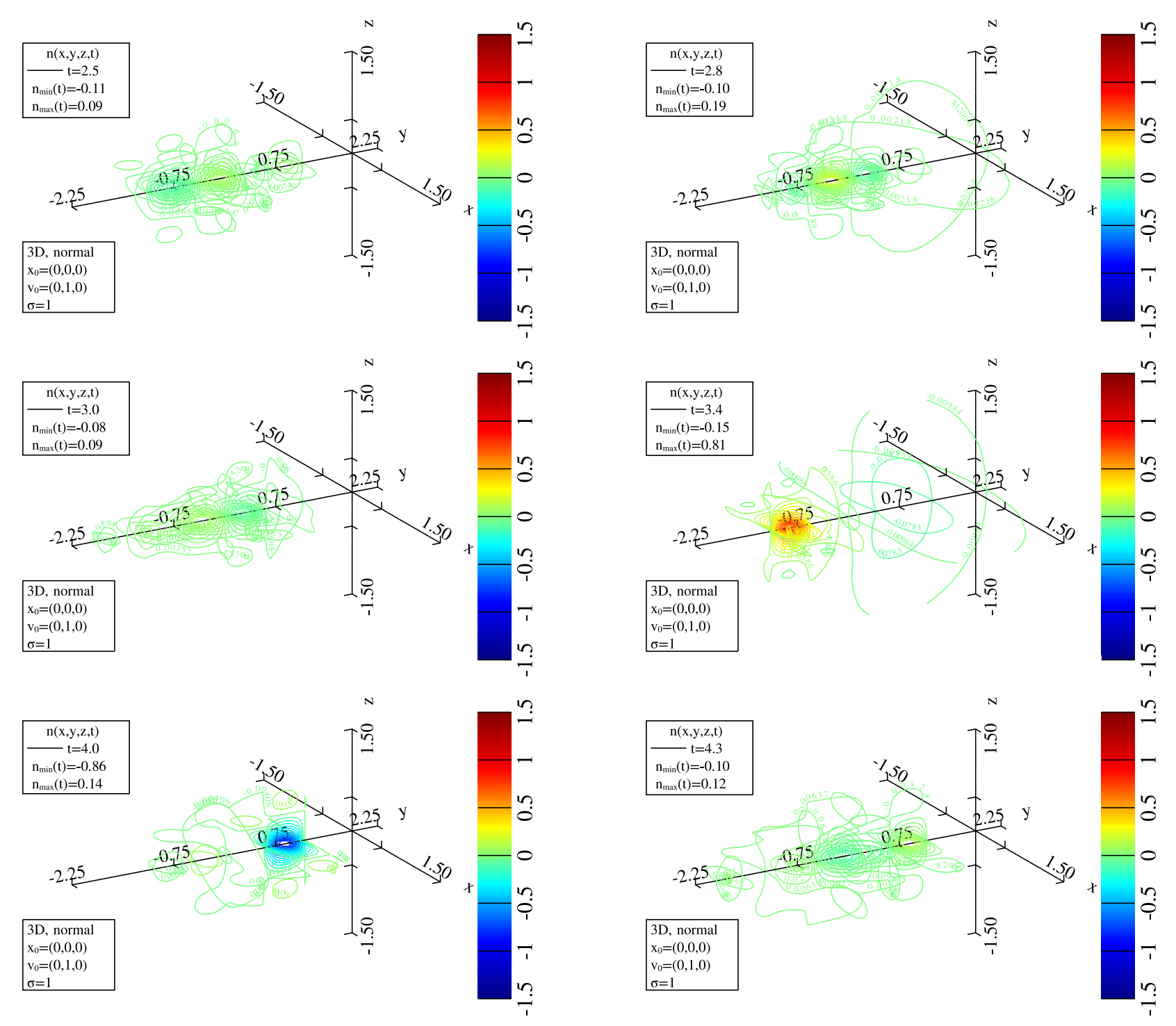

FIG. 9. Dynamics of the density $n(\vec{x}, t)$ for the 3D plasma with normal spatial and velocity distributions for different values of time; the external charge starts moving from $\vec{x}_{0}=(0.0,0.0,0.0)$ with velocity $\vec{v}_{0}=(0.0,1.0,0.0)$.

external charge and the negative density is spread around. This peak reaches its maximum height at $\mathrm{t} \approx 0.9$. Then, this peak continues to exist, and its height decreases, until $\mathrm{t}=1.7$, when it almost disappears and negative peak appears in the negative part of $y$ axis. For $t=2.1$, both peaks are relatively small and have almost the same sizes. Thereafter, for $t=2.3$, we see the negative peak in a negative part of the $y$ axis; for $t=2.5$, the negative and positive peaks are of the same size. Then, a positive peak appears in the negative part of the $y$ axis, and then, the positive and negative peaks have almost same size for $t=3.0$. For $t=3.4$ and $t=4.0$, we see a quite large positive peak in the negative part and negative peak in the positive part, respectively. For $t=4.3$, the peaks are almost of the same size. At this time, the charge is outside of the plasma and barely affects its charge distribution. For larger time values, the oscillations continue but with decreasing amplitudes, as we see for the time values $t=3.4$ and $\mathrm{t}=4.0$, similarly to the lower dimensional cases; for brevity, we do not show these plots.

In this subsection, we described our results for the test charge problem in the 3D confined plasma. These results can be used to analyze the dynamics of shielding of a charged particle in a bunched electron beam that is needed to model the modulator section of the coherent electron cooling; we will discuss this after explaining physics of the observed effects.

\section{Discussion}

In this subsection, we will discuss physical explanation of the numerical results described in previous subsections. Dynamics of the density perturbation caused by interaction of plasma with a moving charged particle has some qualitative features that can be seen in both confined 
and infinite plasmas. However, there are also differences, which are caused by an interaction with the confining fields.

There is always a main positive peak, which follows the charge, as shown in Figs. 3 and 4 for the infinite plasma, and in Figs. 5-9 for the confined; this peak is caused by attraction between plasma's particles and an external charge. In the infinite plasma (with infinite charge), the charges are coming from infinity and sole positive peaks can occur for small time values, as shown in Figs. 3 and 4. In contrast, in the finite plasma, the charge is finite and conserved, and positive peaks are always surrounded by negative density regions or well-formed negative peaks, as shown in Figs. 5-9.

For large time values, negative and spurious positive peaks appear in the infinite plasma. For the infinite 1D Cauchy plasma, appearance of additional peaks is confirmed by the exact analytical solution (116) depicted in Fig. 3, for other distributions, it is confirmed by numerical solutions [6]. These are the plasma oscillations (also known as Langmuir waves) induced by sudden appearance and movement of the external charge. These plasma waves are solutions of the Vlasov-Poisson system for both infinite and confined plasmas and they are also seen in Figures 5-9 for the confined plasma. Their amplitude decreases because of the Landau damping.

The confined plasma considered does not have an actual boundary, however, the confining fields serve as one and we observe reflections of the plasma waves from this effective boundary. In the 1D case, there is only one spatial dimension and reflection of the wave from the boundary leads to decrease of the positive peak and then to its transformation into the negative one. It is seen in Fig. 5: the perturbation reaches its maximum at $t=2.0$ and then decreases, then the charge leaves the plasma and we see standing wave with decreasing amplitude. In Fig. 6, the situation is more complicated, since the charge spends more time in the plasma and travels across it, but reflections are also there. In the $2 \mathrm{D}$ and $3 \mathrm{D}$ cases, the situation is different, since we still have a point (zero-dimensional) charge traveling along a line, which is a $1 \mathrm{D}$ object, but the plasma has more spatial dimensions. The plasma waves sourced at the location of the charge travel to the boundary in all directions, then reflect from the boundary and form a negative peak at the location opposite to the location of the charge with respect to center of the plasma. In the $2 \mathrm{D}$ and $3 \mathrm{D}$ cases, these negative peaks appear at $\mathrm{t}=1.2$ and $\mathrm{t}=1.7$, respectively. Thus, confining fields serve as a reflecting boundary, which focuses reflected waves to the position opposite to the location of the initial peak with respect to the origin in the $2 \mathrm{D}$ and $3 \mathrm{D}$ cases, and just reflects the wave in the $1 \mathrm{D}$ case.

Then, in all confined plasmas, we see a standing density waves. These waves are harmonic oscillations of the perturbation's density in the confining fields, which correspond to $\mathrm{X}$-dependent part of the Hamiltonian $\mathrm{H}_{0}(66)$ :

$$
\mathrm{H}_{0}=\sum_{i} \mathrm{a}_{i} \mathrm{x}_{i}^{2}+\frac{1}{2} \sum_{i} \mathrm{v}_{i}^{2} .
$$

We consider symmetric case, for which all $a_{i}$ equal 1 ; denoting them as $a$, we obtain for the dimensionless frequency $\tilde{\omega}_{0}$ :

$$
\tilde{\omega}_{0}=\sqrt{2 \mathrm{a}}
$$

For $\mathrm{a}=1$, we have for the dimensionless period $\mathrm{T}_{0}$ :

$$
\mathrm{T}_{0}=\frac{2 \pi}{\tilde{\omega}_{0}}=\sqrt{2} \pi \approx 4.44, \quad \text { and } \quad \frac{\mathrm{T}_{0}}{2} \approx 2.22 .
$$

This value for the half-period is in an agreement with the observed behavior. In the $2 \mathrm{D}$ case, the changes of the density profiles between $t=1.8$ and $t=3.9$, and $t=1.2$ and $\mathrm{t}=3.5$ correspond to half-oscillation and time differences are close to the computed value for the half-period. (We plotted densities with time step 0.1, so the exact equality should not be expected.) Similarly, in the $3 \mathrm{D}$ case, we see half-oscillation from $\mathrm{t}=1.7$ to $t=4.0$, and from $t=1.2$ to $t=3.4$. Since the $1 \mathrm{D}$ plasma is not symmetric with respect to charge's trajectory, as the $2 \mathrm{D}$ and $3 \mathrm{D}$ plasmas, and there is no focusing in the 1D case, the standing wave behaves a bit differently in the 1D plasma case.

In the 2D and 3D case, there is another interesting effect: oscillations of the shape of the peaks of the standing density wave. In the $2 \mathrm{D}$ case, we see wide peaks for $\mathrm{t}=1.8$ and $\mathrm{t}=3.9$ and spiky peaks for $\mathrm{t}=1.2$ and $\mathrm{t}=3.5$. Similarly, in the $3 \mathrm{D}$ case, we see wide peaks for $t=2.1$ and $t=4.3$ and spiky peaks for $t=1.7$ and $t=4.0$. This effect is caused by reflection and focusing of the standing wave in the confining fields: the wide density profiles reach the boundary and then focuses into spiky peaks, then widen again, etc. In the 1D case, we do not see this widening and shrinking of the peaks, since we do not have focusing, which is responsible for this effect, the wave just reflects.

Another feature is damped oscillations of the maximum of the positive peak for the large time values. This effect is caused by the Landau damping and confirmed for the infinite plasma by the exact solution (116) plotted in Fig. 3. For the large time values, the charge does not leave the plasma and perturbation continues to exist approaching constant nonzero value at infinity [5]. In the confined plasma case, oscillations in the confining fields have much larger amplitude and this effect is not clearly observable. For the large time values, the charge is outside of the plasma and does not affect much the plasma's charge distribution, which is controlled by the space charge fields and confining fields, which damp the perturbation caused by the charge. 


\section{E. Application to the coherent electron cooling}

The device for the coherent electron cooling proof of principle (PoP) experiment is being constructed in the Relativistic Heavy Ion Collider (RHIC) at Brookhaven National Laboratory [4]. To apply our results to the modulator section of the device, we need to recover the dimensional quantities. For the PoP experiment, we have the Debye radius $r_{\mathrm{D}}=4.65 \times 10^{-5} \mathrm{~m}$ and plasma frequency $\omega_{\mathrm{p}}=6.436 \times 10^{9} \mathrm{~s}^{-1}$. The dimensional electron density perturbation can be obtained as follows:

$$
n_{1}(\vec{x}, t)=r_{\mathrm{D}}^{-d} \mathrm{n}_{1}\left(r_{\mathrm{D}}^{-1} \vec{x}, \omega_{\mathrm{p}} t\right),
$$

where $d$ is a spatial dimension of the problem; for the real 3D case, $d=3$. In the PoP experiment, the modulator is designed such that the interaction time is around one half of the plasma period, it is one half in our dimensionless units, and it depends on the hadron's velocity, since the modulator's length is constant. The velocity is measured in units of electron beam's $v_{\text {rms }}$, in the PoP experiment, $v_{\text {rms }}=3.0 \times 10^{5} \frac{\mathrm{m}}{\mathrm{s}}$.

Our computations for the 3D confined plasma show that for times around one half of the plasma period the density perturbation follows the charge, and there are no considerable negative peaks, which could cause problems in a $\mathrm{CeC}$ device, since they also would be amplified in an FEL and created electric fields that would undesirably accelerate the hadrons. Since the peaks grow with time and we are interested in largest possible ones, the maximum time, for which there are no negative peaks, sets limit on a possible length of the modulator. Although, it is possible to determine the precise time value, for which negative peaks of a certain size appear, it would not make sense, since the modulator's length is constant and hadrons have different velocities, thus their passing through the modulator takes different times. For the 3D ball that we considered, it is $\mathrm{t} \approx 1.2$, this time is larger than the modulation time in the PoP experiment, thus the modulator can even be extended. Moreover, in the actual device, the bunches are not balllike; their longitudinal size is larger than transversal, and so the hadrons will pass through a much smaller part of the bunch, than in our computations, and these edge effects will not play a role, thus, spurious perturbations will not cause any problems.

Although, we shed some light on the dynamics of the perturbation in the device for the $\mathrm{CeC}$ PoP experiment, the main goal of this article was to develop the method and explore the physics of the shielding in the confined plasma. Further investigation of the processes in the coherent electron cooling is planned.

\section{CONCLUSION}

In the present article, we studied in detail dynamical test charge problem for the confined plasma, which is a realistic model of an electron beam. We developed a novel method for solving this problem. We considered a collisionless single-species electron plasma; the method can be easily generalized to a multi-species plasma. While other known nonsimulation methods are applicable only to the infinite plasma [5,6], our method is developed for the confined plasmas and can be modified to deal with the infinite plasma as well.

The idea underlying the method is to transform the Vlasov-Poisson system of differential equations to an integral equation for the Laplace image of the density perturbation, and then to solve this equation numerically. To solve the integral equation, we applied the piecewise polynomial collocation method [14] that we reviewed in detail. We used the FFT to compute the inverse Laplace transform.

We developed a stand-alone solver for the Fredholm integral equations, and for the Laplace-Fredholm ones, which are Fredholm equations for the Laplace image of the quantity of interest. We used it to solve the test charge problem. Our solver can be used to solve other equations of this type; for example, such equations appear in the threedimensional theory of high-gain free-electron lasers [17]. All our programs are parallel and object-oriented; the complete source code is available at [15] under the GNU General Public License; all computations were performed on Cray supercomputers at the National Energy Research Scientific Computing Center (NERSC).

Our computations revealed a few important features of the shielding of a charged particle in a confined plasma. Similarly to the case of the infinite plasma, in addition to the main positive peak, we see plasma oscillations, i.e., negative and positive peaks of the density perturbation, these peaks can be comparable in size to the main positive peak. For the case of confined plasma, especially, for large time values, the shape of the perturbation is mostly determined by the symmetry of the plasma, and we observe density oscillations in the confining fields that occur around zero, while for the case of the infinite plasma [5,6], the density oscillates around some saturation value and follows the charge even for large time values. These differences are caused by nonzero confining and space-charge fields in the confined plasma and by the finiteness of its charge. The confining fields create an effective boundary, which reflects plasma waves. For the 2D and 3D plasmas, we observe focusing of these reflected waves and formation of additional peaks via this mechanism; reflections and focusing also explain oscillations of the shape of the peaks, i.e., spiky and wide peaks are observed. In the 1D plasma, there is no focusing, only reflections, and there are no spiky peaks.

The method developed provides an opportunity to compute shielding effects in confined plasmas, which are realistic models of the electron beams in accelerators. In particular, these methods will allow to advance further theoretical modeling of the coherent electron cooling. 


\section{ACKNOWLEDGMENTS}

Authors are thankful to Gang Wang, George Bell, and Alexei Fedotov for thoughtful discussions of the topic and directing us toward some important previous works. We thank the anonymous referee, whose suggestion helped to improve the article. This research used resources of the National Energy Research Scientific Computing Center, which is supported by the Office of Science of the U.S. Department of Energy under Contract No. DE-AC0205CH11231. This work is supported by the U.S. Department of Energy under Contract No. DE-AC0298CH10886 and by the NSF under Grant PHY-1415252. We thank A. Woodhead for proofreading.

\section{APPENDIX: NUMERICAL EVALUATION OF THE INVERSE LAPLACE AND FOURIER TRANSFORMS}

Here, we describe how the inverse Laplace transform can be computed numerically using the fast Fourier transform (FFT). We refer to [6] for more details and numerical tests. We use the following definition of the Fourier transform:

$$
\mathcal{F} f(x) \equiv \tilde{f}(k)=\int_{-\infty}^{\infty} f(x) \mathrm{e}^{-i k x} d x,
$$

and its inverse:

$$
\mathcal{F}^{-1} \tilde{f}(k) \equiv f(x)=\frac{1}{2 \pi} \int_{-\infty}^{\infty} \tilde{f}(k) \mathrm{e}^{i k x} d k .
$$

The discrete Fourier transform (DFT) assigns to the set of points $\left\{x_{n}\right\}_{0 \leq n \leq N-1}$ the set of points $\left\{X_{k}\right\}_{0 \leq k \leq N-1}$ :

$$
X_{k}=\sum_{n=0}^{N-1} x_{n} \mathrm{e}^{-2 \pi i \frac{k n}{N}}
$$

we use the following notation:

$$
X_{k}=\operatorname{DFT}_{k}\left[\left\{x_{n}\right\}_{0 \leq n \leq N-1}\right] .
$$

The Laplace transform is defined as follows:

$$
\mathcal{L} f(t) \equiv \tilde{f}(s)=\int_{0}^{\infty} f(t) \mathrm{e}^{-t s} d t,
$$

and the inverse:

$$
\mathcal{L}^{-1} \tilde{f}(s) \equiv f(t)=\frac{1}{2 \pi i} \int_{\sigma-i \infty}^{\sigma+i \infty} \tilde{f}(s) \mathrm{e}^{t s} d s
$$

where $\sigma$ is a real constant greater than real parts of all singularities of $\tilde{f}(s)$.
The inverse Fourier transform can be evaluated via the discrete one. Approximating the Fourier integral (A2) by

$$
f(x) \approx \frac{1}{2 \pi} \int_{-a}^{a} \tilde{f}(k) \mathrm{e}^{i k x} d k,
$$

and, introducing the following notations

$k_{n}=-a+\frac{2 a n}{N}, \quad x_{j}=\frac{N \pi}{2 a}-\frac{\pi j}{a}, \quad C_{j}=\frac{a(-1)^{j}}{N \pi} \mathrm{e}^{-i \pi \frac{N}{2}}$,

we obtain

$$
\begin{aligned}
f\left(x_{j}\right) & \approx C_{j} \sum_{n=0}^{N-1} \tilde{f}\left(k_{n}\right)(-1)^{n} \mathrm{e}^{-i \frac{2 \pi n j}{N}} \\
& =C_{j} \operatorname{DFT}_{j}\left[\left\{\tilde{f}\left(k_{n}\right)(-1)^{n}\right\}_{0 \leq n \leq N-1}\right] .
\end{aligned}
$$

The inverse Laplace transform can be expressed via the Fourier transform as follows:

$\mathcal{L}^{-1} \tilde{f}(s)=\frac{\mathrm{e}^{\sigma t}}{2 \pi} \int_{-\infty}^{\infty} \tilde{f}(\sigma+i k) \mathrm{e}^{i k t} d k=\mathrm{e}^{\sigma t} \mathcal{F}_{k}^{-1} \tilde{f}(\sigma+i k)$,

where $\sigma$ is the same as in (A6) and subscript $k$ stands for the fact that the transform is over $k$. Evaluating the inverse Fourier transform via (A10), we obtain:

$f\left(t_{j}\right)=\mathrm{e}^{\sigma t_{j}} C_{j} \operatorname{DFT}_{j}\left[\left\{\tilde{f}\left(\sigma+i k_{n}\right)(-1)^{n}\right\}_{0 \leq n \leq N-1}\right]$.

The discrete Fourier transforms in (A10) and (A12) can be computed numerically using the FFT.

[1] V. N. Litvinenko and Y. S. Derbenev, Phys. Rev. Lett. 102, 114801 (2009).

[2] S. Webb, G. Wang, and V. Litvinenko, Phys. Rev. ST Accel. Beams 14, 051003 (2011).

[3] A. Elizarov and V. Litvinenko, in Proceedings of the Free Electron Laser Conference 2013 (JACoW, New York, USA, 2013), mopso17, http://accelconf.web.cern.ch/ AccelConf/FEL2013/papers/mopso17.pdf.

[4] I. Pinyaev et al., in Proceedings of the 3rd International Particle Accelerator Conference, New Orleans, LA, 2012 (IEEE, Piscataway, NJ, 2012), p. 400, moppd016, http://accelconf.web.cern.ch/accelconf/IPAC2012/papers/ moppd016.pdf; in Proceedings of the 4th International Particle Accelerator Conference, IPAC-2013, Shanghai, China, 2013 (JACoW, Shanghai, China, 2013), p. 1535, tupfi081, http://accelconf.web.cern.ch/AccelConf/ IPAC2013/papers/tupfi081.pdf.

[5] G. Wang and M. Blaskiewicz, Phys. Rev. E 78, 026413 (2008). 
[6] A. Elizarov and V. Litvinenko, Phys. Rev. ST Accel. Beams 16, 124001 (2013).

[7] A. J. Turski, Ann. Phys. (N.Y.) 35, 240 (1965).

[8] R. L. Gluckstern, in Proceedings of the Linac Conference, 1970 (Fermilab, Batavia, IL, 1970), p. 811.

[9] M. Venturini and R. L. Gluckstern, Phys. Rev. ST Accel. Beams 3, 034203 (2000).

[10] R. Tegeback, A. S. Usenko, I. P. Yakimenko, and A. G. Zagorodny, J. Plasma Phys. 18, pp. 113 (1977).

[11] A. A. Vlasov, Sov. Phys. Usp. 10, 721 (1968).
[12] D. R. Nicholson, Introduction to Plasma Theory (Wiley, New York, 1983).

[13] M. Abramowitz and I. Stegun, Handbook on Mathematical Functions (Dover, New York, 1965).

[14] G. Vainikko, Multidimensional Weakly Singular Integral Equations (Springer, New York, 1993).

[15] http://muline.sourceforge.net/.

[16] L. D. Landau and E. M. Lifshitz, Electrodynamics of Continuous Media (Pergamon Press, New York, 1984).

[17] Z. Huang and K.-J. Kim, Phys. Rev. E 62, 7295 (2000). 